\title{
MULTILEVEL MONTE CARLO FINITE VOLUME METHODS FOR RANDOM CONSERVATION LAWS WITH DISCONTINUOUS FLUX
}

\author{
Jayesh Badwaik ${ }^{1}$, Christian Klingenberg ${ }^{1}$, Nils Henrik Risebro ${ }^{2}$ \\ AND Adrian M. RuF ${ }^{3, *}$
}

\begin{abstract}
We consider conservation laws with discontinuous flux where the initial datum, the flux function, and the discontinuous spatial dependency coefficient are subject to randomness. We establish a notion of random adapted entropy solutions to these equations and prove well-posedness provided that the spatial dependency coefficient is piecewise constant with finitely many discontinuities. In particular, the setting under consideration allows the flux to change across finitely many points in space whose positions are uncertain. We propose a single- and multilevel Monte Carlo method based on a finite volume approximation for each sample. Our analysis includes convergence rate estimates of the resulting Monte Carlo and multilevel Monte Carlo finite volume methods as well as error versus work rates showing that the multilevel variant outperforms the single-level method in terms of efficiency. We present numerical experiments motivated by two-phase reservoir simulations for reservoirs with varying geological properties.
\end{abstract}

Mathematics Subject Classification. 35L65, 35R05, 65C05, 65M12.

Received September 25, 2020. Accepted March 9, 2021.

\section{INTRODUCTION}

This paper concerns uncertainty quantification for conservation laws with discontinuous flux of the form

$$
\begin{gathered}
u_{t}+f(k(x), u)_{x}=0, \quad x \in \mathbb{R}, t>0, \\
u(x, 0)=u_{0}(x), \quad x \in \mathbb{R} .
\end{gathered}
$$

Here, $u: \mathbb{R} \times[0, \infty) \rightarrow \mathbb{R}$ is the unknown and $f \in \mathcal{C}^{2}\left(\mathbb{R}^{2} ; \mathbb{R}\right)$ is the flux function having a possibly discontinuous spatial dependency through the coefficient $k$. In particular, we will assume that the initial datum $u_{0}$ is in $\left(\mathrm{L}^{\infty} \cap \mathrm{BV}\right)(\mathbb{R})$, the flux $f$ is strictly increasing in $u$, and the coefficient $k$ is piecewise constant with finitely many discontinuities. Going back to (1.1), this amounts to switching from one $u$-dependent flux to another across finitely many points in space.

Keywords and phrases. Uncertainty quantification, conservation laws, discontinuous flux, numerical methods.

1 Department of Mathematics, University of Würzburg, Würzburg, Germany.

2 Department of Mathematics, University of Oslo, Oslo, Norway.

3 Seminar for Applied Mathematics, ETH Zürich, Switzerland.

* Corresponding author: adrian.ruf@sam.math.ethz.ch 
Equations of type (1.1) arise in a number of areas of application including vehicle traffic flow in the presence of abruptly varying road conditions (see [35]), polymer flooding in oil recovery (see [48]), two-phase flow through heterogeneous porous media (see $[22,23,44]$ ), and sedimentation processes (see $[9,14]$ ).

Even in the absence of flux discontinuities, and even if the initial datum is smooth, solutions of (1.1) develop discontinuities in finite time and for this reason weak solutions are sought. Weak solutions to (1.1) are not unique, so the weak formulation of the problem is augmented with an additional entropy condition. In the case where $x \mapsto f(k(x), u)$ is smooth, uniqueness follows from the classical Kružkov entropy conditions [33]. In the presence of spatial flux discontinuities, standard Kružkov entropy conditions no longer make sense. This difficulty is usually resolved by requiring that Kružkov entropy conditions hold away from the spatial flux discontinuities and imposing additional jump conditions along the spatial interfaces $[1,4,5,14,21,22,26,29,30,49,50]$ or by adapting the Kružkov entropy conditions in a suitable way $[6-8,42,46,51]$. In the present paper we will focus on the second approach of so-called adapted entropy solutions for which we need to require that the flux function $f$ is strictly monotone in $u$.

In the last two decades, there has been a large interest in the numerical approximation of entropy solutions of (1.1) under various assumptions on $k$ and $f$. We refer to $[3,4,9,10,12,20-22,25-28,30,31,37,49,50,55]$ for a partial list of references regarding finite volume methods respectively the front tracking method. Specifically, in the adapted entropy framework we want to highlight the results of $[7,8,15-17,42,46,51]$ regarding finite volume methods and the front tracking method. We refer to $[5,7,46]$ for an overview of the literature concerning conservation laws with discontinuous flux.

The classical paradigm for designing efficient numerical schemes assumes that data for (1.1), i.e., the initial datum $u_{0}$, the flux $f$, and the spatial dependency coefficient $k$, are known exactly.

However, in many situations of practical interest, there is an inherent uncertainty in the modeling and measurement of physical parameters. For example, in two-phase flow through a heterogeneous porous medium the position of the interface between two rock types is typically not known exactly. Often these parameters are only known up to certain statistical quantities of interest like the mean, variance, or higher moments. In such cases, a mathematical framework of (1.1) is required which allows for random data.

For standard conservation laws without spatial flux dependency, i.e., for

$$
\begin{array}{ll}
u_{t}+f(u)_{x}=0, & x \in \mathbb{R}, t>0, \\
u(x, 0)=u_{0}(x), & x \in \mathbb{R},
\end{array}
$$

such a framework was developed in a series of papers allowing for random initial datum [38], random (spatially independent) flux [41], and even random source terms [39] and random diffusion [32].

The first aim of the current paper is to extend this mathematical framework to include scalar conservation laws with discontinuous flux with random discontinuous spatial dependency. To that end, we define random entropy solutions and provide an existence and uniqueness result, which generalizes the well-posedness results for (1.2) to the case of uncertain initial datum, flux, and discontinuous spatial dependency. In particular, our framework allows for uncertain positions of the flux discontinuities.

The second aim of this paper is to design fast and robust numerical algorithms for computing the mean of random entropy solutions of conservation laws with discontinuous flux. Specifically, we propose and analyze a multilevel combination of Monte Carlo (MC) sampling and a "pathwise" finite volume method (FVM) to approximate the mean of random entropy solutions of conservation laws with discontinuous flux. The multilevel Monte Carlo finite volume method (MLMCFVM) for (1.1) is non-intrusive (in the sense that it requires only repeated applications of existing solvers for input data samples), easy to implement and to parallelize, and well suited for random solutions with low spatial regularity. Solutions exhibiting spatial discontinuities are generic for conservation laws and, in particular, for conservation laws with discontinuous flux. This reduced regularity poses some challenges to the design of efficient so-called stochastic Galerkin methods for example which are based on generalized polynomial chaos. These methods are well-developed for conservation laws - albeit without flux discontinuities - (see $[2,11,36,43,52,54]$ and references therein), but they are more intrusive, generally harder to 
implement and to parallelize. Thus, in the present paper, we focus on the design and mathematical analysis of statistical MC-type methods. Our analysis includes the proof of convergence rates at which the MCFVM and the MLMCFVM converge towards the mean of the random entropy solution of (1.1). The analysis is complicated by the fact that adapted entropy solutions of (1.1) do not possess the same stability properties as entropy solutions of (1.2). Moreover, we determine the number of MC samples needed to minimize the computational work for a given error tolerance.

We want to emphasize that the framework of adapted entropy solutions and more specifically the setting of the present paper is currently the only setting for which we simultaneously have existence [51], uniqueness [6], stability with respect to the modeling parameters [46], and numerical methods with a provable convergence rate $[7,46]$ - the essential components for an uncertainty quantification framework (cf. [41]).

The remainder of this paper is organized as follows. In Section 2 we introduce preliminary results regarding the MC approximation of Banach space-valued random variables. Section 3 is devoted to a review of existence and stability results regarding entropy solutions of (deterministic) conservation laws with discontinuous flux of the form (1.1). In Section 4 we introduce random entropy solutions of (1.1) where the initial datum $u_{0}$, the flux $f$, and the discontinuous coefficient $k$ are subject to randomness. In particular, we prove existence and uniqueness of random entropy solutions. In Section 5, we first review a FVM which was introduced in [7] for the deterministic problem, prove certain stability estimates, and then extend the FVM to MC as well as MLMC versions for (1.1) with random parameters. In Section 6 we perform numerical experiments motivated by two-phase reservoir simulations for reservoirs with varying geological properties to validate our error estimates. Finally, we summarize the findings of this paper in Section 7.

\section{Preliminaries on the Monte Carlo method}

We first introduce some preliminary concepts which are needed in the exposition. To that end, we follow $[34,53]$, see also Section 2 of [32] and Section 5 of [13].

Given a probability space $(\Omega, \mathcal{F}, \mathbb{P})$, a Banach space $V$, and a random variable $X: \Omega \rightarrow V$ we are interested in approximating the mean $\mathbb{E}[X]$ of $X$ via Monte Carlo sampling. To this end, let $\left(\hat{X}^{i}\right)_{i=1}^{M}, i=1, \ldots, M$, be $M$ independent, identically distributed samples of $X$. Then, the Monte Carlo estimator $E_{M}[X]$ of $\mathbb{E}[X]$ is defined as the sample average

$$
E_{M}[X]:=\frac{1}{M} \sum_{i=1}^{M} \hat{X}^{i} .
$$

We are interested in deriving a rate at which

$$
\left\|\mathbb{E}[X]-E_{M}[X]\right\|_{L^{q}(\Omega ; V)}=\mathbb{E}\left[\left\|\mathbb{E}[X]-E_{M}[X]\right\|_{V}^{q}\right]^{\frac{1}{q}}
$$

converges as $M \rightarrow \infty$ for some $1 \leq q<\infty$ and some Banach space $V$ (typically a Lebesgue space). For general Banach spaces $V$ such convergence rate estimates depend on the type of the Banach space.

Definition 2.1 (Banach space of type $q$ [34], p. 246). Assume that $\Omega$ permits a sequence of independent Rademacher random variables $Z_{i}, i \in \mathbb{N}$. We say that a Banach space $V$ is a Banach space of type $1 \leq q \leq 2$ if there is a constant $\kappa>0$ such that for all finite sequences $\left(x_{i}\right)_{i=1}^{M} \subseteq V$

$$
\left(\mathbb{E}\left\|\sum_{i=1}^{M} Z_{i} x_{i}\right\|_{V}^{q}\right)^{\frac{1}{q}} \leq \kappa\left(\sum_{i=1}^{M}\left\|x_{i}\right\|_{V}^{q}\right)^{\frac{1}{q}} .
$$

We will refer to $\kappa$ as the type constant of $V$. 
Every Banach space is a Banach space of type 1 and every Hilbert space a Banach space of type 2 ([34], Thm. 9.10). Moreover, $\mathrm{L}^{p}$ spaces are Banach spaces of type $q=\min (2, p)$ for $1 \leq p<\infty$ ([34], p. 247). We will need the following results regarding Lebesgue spaces of functions with values in a Banach space of type $q$.

Lemma 2.2 ([34], p. 247). Let $1 \leq r \leq \infty,(\Omega, \mathcal{F}, \mathbb{P})$ be a measure space, and $V$ be a Banach space of type $q$. Then the space $\mathrm{L}^{r}(\Omega, V)$ is a Banach space of type $\min (r, q)$.

Proposition 2.3 ([34], Prop. 9.11). Let $V$ be a Banach space of type $q$ with type constant $\kappa$. Then, for every finite sequence $\left(X_{i}\right)_{i=1}^{M}$ of independent mean zero random variables in $\mathrm{L}^{q}(\Omega, V)$, we have

$$
\mathbb{E}\left[\left\|\sum_{i=1}^{M} X_{i}\right\|_{V}^{q}\right] \leq(2 \kappa)^{q} \sum_{i=1}^{M} \mathbb{E}\left[\left\|X_{i}\right\|_{V}^{q}\right] .
$$

Corollary 2.4 ([32], Cor. 2.5). Let $V$ be a Banach space of type $q$ with type constant $\kappa$ and let $X \in \mathrm{L}^{q}(\Omega ; V)$ be a zero mean random variable. Then for every finite sequence $\left(X_{i}\right)_{i=1}^{M}$ of independent, identically distributed random variables with zero mean and with $X_{i} \stackrel{D}{=} X$, we have

$$
\mathbb{E}\left[\left\|E_{M}[X]\right\|_{V}^{q}\right]=\mathbb{E}\left[\left\|\frac{1}{M} \sum_{i=1}^{M} X_{i}\right\|_{V}^{q}\right] \leq(2 \kappa)^{q} M^{1-q} \mathbb{E}\left[\|X\|_{V}^{q}\right] .
$$

We can use Corollary 2.4 to derive a convergence rate of the Monte Carlo estimator in $\mathrm{L}^{q}\left(\Omega ; \mathrm{L}^{p}(\mathbb{R})\right)$ for random variables in $\mathrm{L}^{r}\left(\Omega ; \mathrm{L}^{p}(\mathbb{R})\right)$.

Theorem 2.5. Let $1 \leq r, p \leq \infty$ and $X \in \mathrm{L}^{r}\left(\Omega ; \mathrm{L}^{p}(\mathbb{R})\right)$, then for $q:=\min \{2, p, r\}$ we have the Monte Carlo error estimate

$$
\left\|\mathbb{E}[X]-E_{M}[X]\right\|_{L^{q}\left(\Omega ; \mathrm{L}^{p}(\mathbb{R})\right)} \leq C M^{\frac{1-q}{q}}\|X\|_{\mathrm{L}^{q}\left(\Omega ; \mathrm{L}^{p}(\mathbb{R})\right)} .
$$

In particular, if $p, r>1$ (and thus $q>1$ ) the Monte Carlo estimator $E_{M}[X]$ converges towards $\mathbb{E}[X]$ in $\mathrm{L}^{q}\left(\Omega ; \mathrm{L}^{p}(\mathbb{R})\right)$.

The proof of this theorem is an adaptation of Theorem 4.1 from [32].

Proof. We have

$$
\begin{aligned}
\left\|\mathbb{E}[X]-E_{M}[X]\right\|_{L^{q}\left(\Omega ; \mathrm{L}^{p}(\mathbb{R})\right)}^{q} & =\mathbb{E}\left[\left\|\mathbb{E}[X]-\frac{1}{M} \sum_{i=1}^{M} \hat{X}^{i}\right\|_{\mathrm{L}^{p}(\mathbb{R})}^{q}\right] \\
& =\mathbb{E}\left[\left\|\frac{1}{M} \sum_{i=1}^{M}\left(\mathbb{E}[X]-\hat{X}^{i}\right)\right\|_{L^{p}(\mathbb{R})}^{q}\right] .
\end{aligned}
$$

If we define $Y=\mathbb{E}[X]-X$ and $Y_{i}=\mathbb{E}[X]-\hat{X}^{i}$ we see that $Y$ is in $\mathrm{L}^{r}\left(\Omega ; \mathrm{L}^{p}(\mathbb{R})\right)$ with zero mean and $Y_{i}$ are i.i.d. random variables with zero mean satisfying $Y_{i} \stackrel{D}{=} Y$. Therefore, we can apply Corollary 2.4 since $\operatorname{L}^{r}\left(\Omega ; \mathrm{L}^{p}(\mathbb{R})\right)$ is of type $\min (2, r, p)$ and $\mathrm{L}^{p}(\mathbb{R})$ is of type $\min (2, p)$ and thus in particular also of type $\min (2, r, p)$. Hence,

$$
\mathbb{E}\left[\left\|\frac{1}{M} \sum_{i=1}^{M}\left(\mathbb{E}[X]-\hat{X}^{i}\right)\right\|_{L^{p}(\mathbb{R})}^{q}\right] \leq(2 \kappa)^{q} M^{1-q} \mathbb{E}\left[\|\mathbb{E}[X]-X\|_{L^{p}(\mathbb{R})}^{q}\right]
$$


where $\kappa$ is the type constant of $\mathrm{L}^{p}(\mathbb{R})$. It remains to show $\mathbb{E}\left[\|\mathbb{E}[X]-X\|_{\mathrm{L}^{p}(\mathbb{R})}^{q}\right] \leq C \mathbb{E}\left[\|X\|_{\mathrm{L}^{p}(\mathbb{R})}^{q}\right]$. This follows from standard estimates and Jensen's inequality in the following way:

$$
\begin{aligned}
\mathbb{E}\left[\|\mathbb{E}[X]-X\|_{\mathrm{L}^{p}(\mathbb{R})}^{q}\right] & \leq C \mathbb{E}\left[\|\mathbb{E}[X]\|_{\mathrm{L}^{p}(\mathbb{R})}^{q}+\|X\|_{\mathrm{L}^{p}(\mathbb{R})}^{q}\right] \\
& \leq C\left(\left(\mathbb{E}\left[\|X\|_{\mathrm{L}^{p}(\mathbb{R})}\right]\right)^{q}+\mathbb{E}\left[\|X\|_{\mathrm{L}^{p}(\mathbb{R})}^{q}\right]\right) \\
& \leq C \mathbb{E}\left[\|X\|_{\mathrm{L}^{p}(\mathbb{R})}^{q}\right] .
\end{aligned}
$$

Note that Corollary 2.4 and Theorem 2.5 do not imply convergence if $q=1$, i.e., if $r$ or $p$ are equal to 1 in the latter case.

\section{Deterministic Conservation LaWs With Discontinuous Flux}

In this section, we present the main existence and stability results for deterministic conservation laws with spatially discontinuous flux from $[8,46,51]$.

We consider the Cauchy problem for conservation laws with discontinuous flux of the form

$$
\begin{gathered}
u_{t}+f(k(x), u)_{x}=0, \quad x \in \mathbb{R}, t>0 \\
u(x, 0)=u_{0}(x), \quad x \in \mathbb{R} .
\end{gathered}
$$

Here, we require that $f, k$, and $u_{0}$ satisfy the following:

Assumption 3.1. We assume that the flux $f \in \mathcal{C}^{2}\left(\mathbb{R}^{2} ; \mathbb{R}\right)$ is strictly monotone in $u$ in the sense that $f_{u} \geq \alpha>0$, and that $f\left(k^{*}, 0\right)=0$ for all $k^{*} \in \mathbb{R}$. Furthermore, we assume that $k$ is piecewise constant with finitely many discontinuities and that the initial datum $u_{0}$ is in $\left(\mathrm{L}^{\infty} \cap \mathrm{BV}\right)(\mathbb{R})$.

In the deterministic setting, we consider entropy solutions in the following sense $(c f .[6,8])$. For $p \in \mathbb{R}$ we define the function $c_{p}: \mathbb{R} \rightarrow \mathbb{R}$ through the equation

$$
f\left(k(x), c_{p}(x)\right)=p, \quad \text { for all } x \in \mathbb{R} .
$$

Since $f_{u} \geq \alpha>0$ this equation has a unique solution for each $x \in \mathbb{R}$. Note that in the case of piecewise constant $k$ the function $c_{p}$ is piecewise constant as well.

Definition 3.2 (Entropy solution). We say $u \in \mathcal{C}\left([0, T] ; \mathrm{L}^{1}(\mathbb{R})\right) \cap \mathrm{L}^{\infty}((0, T) \times \mathbb{R})$ is an entropy solution of $(3.1)$ if

$$
\begin{aligned}
\int_{0}^{T} \int_{\mathbb{R}}\left(\left|u-c_{p}(x)\right| \varphi_{t}+\operatorname{sgn}\left(u-c_{p}(x)\right)(\right. & \left.\left.f(k(x), u)-f\left(k(x), c_{p}(x)\right)\right) \varphi_{x}\right) \mathrm{d} x \mathrm{~d} t \\
& -\int_{\mathbb{R}}\left|u(x, T)-c_{p}(x)\right| \varphi(x, T) \mathrm{d} x+\int_{\mathbb{R}}\left|u_{0}(x)-c_{p}(x)\right| \varphi(x, 0) \mathrm{d} x \geq 0
\end{aligned}
$$

for all $p \in \mathbb{R}$ and for all nonnegative $\varphi \in \mathcal{C}_{c}^{\infty}(\mathbb{R} \times[0, T])$.

Note that a Rankine-Hugoniot-type argument shows that across a discontinuity $\xi$ of $k$ the entropy solution $u$ satisfies the Rankine-Hugoniot condition

$$
f(k(\xi-), u(\xi-, t))=f(k(\xi+), u(\xi+, t)) \quad \text { for almost every } t \in(0, T)
$$

where $k(\xi \mp)$ and $u(\xi \mp, \cdot)$ denote the left and right traces of $k$ respectively $u$ both of which exist due to Remark 2.3 of [5]. In our subsequent analysis we will rely on the following two results concerning existence and stability of entropy solutions. 
Theorem 3.3 (Existence and uniqueness of entropy solutions [7,8,51]). Let $f, k$, and $u_{0}$ satisfy Assumption 3.1. Then there exists a unique entropy solution $u$ of (3.1) which satisfies

$$
\begin{aligned}
\|u(\cdot, t)\|_{\mathrm{L}^{\infty}(\mathbb{R})} & \leq \frac{C_{f}}{\alpha}\left\|u_{0}\right\|_{\mathrm{L}^{\infty}(\mathbb{R})} \\
\operatorname{TV}(u(\cdot, t)) & \leq C\left(\operatorname{TV}(k)+\operatorname{TV}\left(u_{0}\right)\right)
\end{aligned}
$$

for all $0 \leq t \leq T$ and

$$
\operatorname{TV}_{[0, T]}(u(x, \cdot)) \leq C \operatorname{TV}\left(u_{0}\right)
$$

for all $x \in \mathbb{R}$. Here $C_{f}$ denotes the maximal Lipschitz constant of $f$ and $\alpha$ is as in Assumption 3.1.

Proof. The existence and uniqueness statement follows from the theory developed by Baiti and Jenssen [8]. The $\mathrm{L}^{\infty}$ and TV bounds follow from Theorem 1.4 of [51] and Lemma 4.6 of [7].

Theorem 3.4 (Stability of entropy solutions [46]). Let $f, k$, and $u_{0}$ satisfy Assumption 3.1 and $u$ be the corresponding entropy solution of (3.1). If $v$ is the entropy solution of (3.1) with flux $g$, coefficient $l$, and initial datum $v_{0}$ satisfying Assumption 3.1 then for all $0 \leq t \leq T$

$$
\|u(\cdot, t)-v(\cdot, t)\|_{\mathrm{L}^{1}(\mathbb{R})} \leq\left\|u_{0}-v_{0}\right\|_{\mathrm{L}^{1}(\mathbb{R})}+C\left(\|k-l\|_{\mathrm{L}^{\infty}(\mathbb{R})}+\left\|f_{u}-g_{u}\right\|_{\mathrm{L}^{\infty}\left(\mathbb{R}^{2} ; \mathbb{R}\right)}\right) .
$$

In particular, entropy solutions of (3.1) satisfy

$$
\|u(\cdot, t)\|_{\mathrm{L}^{1}(\mathbb{R})} \leq\left\|u_{0}\right\|_{\mathrm{L}^{1}(\mathbb{R})}
$$

for all $0 \leq t \leq T$.

Proof. The stability estimate can be found in Theorem 4.1 of [46]. The $\mathrm{L}^{1}$ bound follows from the stability estimate (3.4) by taking $g=f, l=k$, and $v_{0}=0$.

Remark 3.5. We want to mention that the stability result from Theorem 3.4 is not only integral in proving existence and uniqueness of random entropy solutions, but can also be used to show well-posedness of Bayesian inverse problems for conservation laws with discontinuous flux [40].

\section{RANDOM CONSERVATION LAWS With DiscontinuOUS FLUX}

We now consider conservation laws with discontinuous flux where the flux $f$, the coefficient $k$, and the initial datum $u_{0}$ in $(3.1)$ are uncertain. To that end, we let $(\Omega, \mathcal{F}, \mathbb{P})$ be a probability space and denote by $\mathcal{B}(X)$ the Borel $\sigma$-algebra on a space $X$. We define appropriate random data $\left(u_{0}, k, f\right)$ in the following sense.

Definition 4.1 (Random data). Given constants $C_{\mathrm{TV}}, C_{f} \in \mathbb{R}, \alpha \in(0, \infty), N_{k} \in \mathbb{Z}, \delta>0$ and given a rectangle $R=R_{1} \times R_{2} \subset \mathbb{R}^{2}$ let $\mathbb{D}$ be the Banach space

$$
\mathbb{D}=\left(\mathrm{BV} \cap \mathrm{L}^{\infty}\right)(\mathbb{R}) \times \mathrm{L}^{\infty}(\mathbb{R}) \times \mathcal{C}^{2}(R ; \mathbb{R})
$$

endowed with the norm

$$
\left\|\left(u_{0}, k, f\right)\right\|_{\mathbb{D}}=\left\|u_{0}\right\|_{L^{1}(\mathbb{R})}+\operatorname{TV}\left(u_{0}\right)+\left\|u_{0}\right\|_{L^{\infty}(\mathbb{R})}+\|k\|_{L^{\infty}(\mathbb{R})}+\|f\|_{\mathcal{C}^{2}(R ; \mathbb{R})} .
$$

We say that a strongly measurable map $\left(u_{0}, k, f\right):(\Omega, \mathcal{F}) \rightarrow(\mathbb{D}, \mathcal{B}(\mathbb{D}))$ is called random data for $(3.1)$ if for $\mathbb{P}$-a.e. $\omega$

$$
\begin{aligned}
u_{0}(\omega ; x) & \in R_{1}, \quad \text { for a.e. } x \in \mathbb{R}, \\
\operatorname{TV}\left(u_{0}\right) \leq C_{\mathrm{TV}} & <\infty,
\end{aligned}
$$




$$
k(\omega ; x) \in R_{2}, \quad \text { for a.e. } x \in \mathbb{R},
$$

$k(\omega ; \cdot)$ is pcw. constant with at most $N_{k}$ discontinuities,

each pair of discontinuities of $k$ is at least $\delta$ apart,

$$
\begin{aligned}
f_{u}(\omega, k, u) \geq \alpha>0 \text { and } f(\omega ; k, 0)=0, & \text { for all }(k, u) \in R, \\
\|f(\omega ; \cdot, \cdot)\|_{\mathcal{C}^{2}(R ; \mathbb{R})} \leq C_{f}<\infty &
\end{aligned}
$$

such that for $\mathbb{P}$-a.e. $\omega$ the data $\left(u_{0}(\omega), k(\omega), f(\omega)\right)$ satisfy Assumption 3.1.

We are interested in random entropy solutions of the random conservation law

$$
\begin{gathered}
\frac{\partial u(\omega ; x, t)}{\partial t}+\frac{\partial f(\omega ; k(\omega ; x), u(\omega ; x, t))}{\partial x}=0, \quad \omega \in \Omega, x \in \mathbb{R}, t>0, \\
u(\omega ; x, 0)=u_{0}(\omega ; x), \quad \omega \in \Omega, x \in \mathbb{R} .
\end{gathered}
$$

Definition 4.2 (Random entropy solution). Given random data $\left(u_{0}, k, f\right): \Omega \rightarrow \mathbb{D}$, we say that a random variable $u: \Omega \rightarrow \mathcal{C}\left([0, T] ; \mathrm{L}^{1}(\mathbb{R})\right)$ is a random entropy solution of (4.1) if $u$ is strongly measurable and for $\mathbb{P}$-a.e. $\omega \in \Omega$ the function $u(\omega)$ satisfies

$$
\begin{aligned}
\int_{0}^{T} \int_{\mathbb{R}}\left(\left|u(\omega ; x, t)-c_{p}(\omega ; x)\right| \varphi_{t}+q(\omega ; u(\omega ; x, t))\right) \mathrm{d} x \mathrm{~d} t \\
\quad-\int_{\mathbb{R}}\left|u(\omega ; x, T)-c_{p}(\omega ; x)\right| \varphi(x, T) \mathrm{d} x+\int_{\mathbb{R}}\left|u_{0}(\omega ; x)-c_{p}(\omega ; x)\right| \varphi(x, 0) \mathrm{d} x \geq 0
\end{aligned}
$$

for all $p \in \mathbb{R}$ and nonnegative $\varphi \in \mathcal{C}_{c}^{\infty}(\mathbb{R} \times[0, T])$. Here we have used the notation

$$
q(\omega ; u(\omega ; x, t))=\operatorname{sgn}\left(u-c_{p}(\omega ; x)\right)\left(f(\omega ; k(\omega ; x), u)-f\left(\omega ; k(\omega ; x), c_{p}(\omega ; x)\right)\right) .
$$

We have the following existence and uniqueness result for random entropy solutions of conservation laws with discontinuous flux.

Theorem 4.3 (Existence and pathwise uniqueness of random entropy solutions). Let $\left(u_{0}, k, f\right)$ be random data. Then there exists a unique random entropy solution $u: \Omega \rightarrow \mathcal{C}\left([0, T] ; \mathrm{L}^{1}(\mathbb{R})\right)$ to (4.1) which is pathwise unique, i.e., if the random data $\left(u_{0}, k, f\right)$ and $\left(v_{0}, l, g\right)$ are $\mathbb{P}$-versions of each other and $u$ and $v$ are corresponding random entropy solutions then $u$ and $v$ are $\mathbb{P}$-versions of each other.

Proof. Let $S: \mathbb{D} \rightarrow \mathcal{C}\left([0, T] ; \mathrm{L}^{1}(\mathbb{R})\right)$ denote the solution operator from Theorem 3.3 that maps (deterministic) $\left(u_{0}, k, f\right) \in \mathbb{D}$ to the unique (deterministic) entropy solution $\hat{u}=S\left(u_{0}, k, f\right)$. Because of the stability estimate (3.4) this solution map is Lipschitz continuous. Now, since the random data $\left(u_{0}, k, f\right): \Omega \rightarrow \mathbb{D}$ is strongly measurable the composition $S \circ\left(u_{0}, k, f\right): \Omega \rightarrow \mathcal{C}\left([0, T] ; \mathrm{L}^{1}(\mathbb{R})\right)$ is again strongly measurable (see [53], Cor. 1.13). Hence $u=S \circ\left(u_{0}, k, f\right)$ is a strongly measurable map satisfying (4.2) $\mathbb{P}$-almost surely. Therefore, $u$ is a random entropy solution to (4.1).

Regarding uniqueness of random entropy solutions, let $\left(u_{0}, k, f\right)$ and $\left(v_{0}, l, g\right)$ be $\mathbb{P}$-versions of each other, i.e., $\left\|\left(u_{0}(\omega), k(\omega), f(\omega)\right)-\left(v_{0}(\omega), l(\omega), g(\omega)\right)\right\|_{\mathbb{D}}=0$ for $\mathbb{P}$-a.e. $\omega \in \Omega$, and $u$ and $v$ corresponding random entropy solutions. Then, the Lipschitz continuity of the solution operator $S$ gives

$$
\|u(\omega)-v(\omega)\|_{\mathcal{C}\left([0, T] ; \mathrm{L}^{1}(\mathbb{R})\right)} \leq C\left\|\left(u_{0}(\omega), k(\omega), f(\omega)\right)-\left(v_{0}(\omega), l(\omega), g(\omega)\right)\right\|_{\mathbb{D}}=0 .
$$

Thus, we have $u(\omega)=v(\omega)$ in $\mathcal{C}\left([0, T] ; \mathrm{L}^{1}(\mathbb{R})\right)$ for $\mathbb{P}$-a.e. $\omega \in \Omega$ which is pathwise uniqueness.

Note that Theorem 4.3 generalizes the existence result of random entropy solutions of [41] for fluxes which are strictly monotone in $u$ since the present setting allows for a discontinuous spatial dependency of the flux. 
Remark 4.4. All existence and continuous dependence results stated so far apply to the deterministic Cauchy problem (3.1). By the usual arguments, verbatim the same results hold for entropy solutions on bounded intervals $D \subset \mathbb{R}$ as well, provided periodic boundary conditions are enforced.

The following probabilistic bound will be important in the numerical approximation of random entropy solutions on bounded domains.

Lemma 4.5. Let $\left(u_{0}, k, f\right)$ be random data and $D \subset \mathbb{R}$ a bounded interval. Let further $u_{0} \in \mathrm{L}^{r}\left(\Omega ; \mathrm{L}^{\infty}(D)\right)$, for some $1 \leq r \leq \infty$. Then the random entropy solution $u$ of $(4.1)$ is in $\mathrm{L}^{r}\left(\Omega ; \mathcal{C}\left([0, T] ; \mathrm{L}^{p}(D)\right)\right)$ for all $1 \leq p \leq \infty$. In particular,

for all $0 \leq t \leq T$.

$$
\|u(\cdot, t)\|_{L^{r}\left(\Omega ; \mathrm{L}^{p}(D)\right)} \leq C\left\|u_{0}\right\|_{\mathrm{L}^{r}\left(\Omega ; \mathrm{L}^{\infty}(D)\right)}
$$

Proof. On bounded domains $D$ we have

$$
\|u(\cdot, t)\|_{\mathrm{L}^{p}(D)} \leq|D|^{\frac{1}{p}}\|u(\cdot, t)\|_{\mathrm{L}^{\infty}(D)}
$$

and thus using the $\mathrm{L}^{\infty}$-bound (3.3) we have for all $0 \leq t \leq T$

$$
\begin{aligned}
\|u(\cdot, t)\|_{\mathrm{L}^{r}\left(\Omega ; \mathrm{L}^{p}(D)\right)}^{r} & =\int_{\Omega}\|u(\cdot, t)\|_{\mathrm{L}^{p}(D)}^{r} \mathrm{~d} \mathbb{P} \\
& \leq C \int_{\Omega}\|u(\cdot, t)\|_{\mathrm{L}^{\infty}(D)}^{r} \mathrm{~d} \mathbb{P} \\
& \leq C \int_{\Omega}\left\|u_{0}\right\|_{\mathrm{L}^{\infty}(D)}^{r} \mathrm{~d} \mathbb{P} \\
& =C\left\|u_{0}\right\|_{\mathrm{L}^{r}\left(\Omega ; \mathrm{L}^{\infty}(D)\right)}^{r}
\end{aligned}
$$

which proves the claim.

\section{NUMERICAL APPROXIMATION OF RANDOM ENTROPY SOLUTIONS}

In this section, we want to approximate the expectation $\mathbb{E}[u(\cdot, t)]$ of a random entropy solution $u$ of the random conservation law with discontinuous flux (4.1). On the one hand, we will use the Monte Carlo and multilevel Monte Carlo method to approximate in the stochastic domain $\Omega$. On the other hand, since in general exact solutions to (4.1) are not at hand, we will approximate in the physical domain $\mathbb{R} \times[0, T]$ by a finite volume method. To this end, we use a modified version of monotone finite volume methods for conservation laws introduced in [7] which appropriately addresses the presence of the discontinuous parameter $k$.

The resulting approximation error introduced by the Monte Carlo method depends on the number of samples used, while the error introduced by the finite volume method depends on the resolution of the grid. In the following subsections, we will review the finite volume method for the deterministic problem, detail how to combine it with the Monte Carlo and multilevel Monte Carlo method and prove error estimates for the resulting Monte Carlo and multilevel Monte Carlo finite volume method.

\subsection{Finite volume methods for conservation laws with discontinuous flux}

We will first consider the (deterministic) conservation law with discontinuous flux (3.1) and present a class of finite volume methods introduced in [7].

For a given (deterministic) function $k$ with discontinuities $\xi_{1}<\xi_{2}<\ldots<\xi_{N}$ such that $k$ satisfies the relevant assumptions in Definition 4.1 we denote by $D_{i}=\left(\xi_{i}, \xi_{i+1}\right), i=0, \ldots, N$, the subdomains where $k$ is constant. Here, we have used the notation $\xi_{0}=-\infty$ and $\xi_{N+1}=+\infty$. In the following we will write

$$
f^{(i)}=f(k(x), \cdot), \quad \text { for } x \in D_{i}, i=0, \ldots, N .
$$


We discretize the domain $\mathbb{R} \times[0, T]$ using the spatial and temporal grid discretization parameters $\Delta x$ and $\Delta t$. Here we assume that the spatial discretization parameter $\Delta x$ is already small with respect to the given minimal distance $\delta$ between discontinuities of $k$, i.e., $\Delta x<\delta$. In order to define the finite volume method we need the spatial grid to be aligned in such a way that all discontinuities of $k$ lie on grid points. We acomplish that in the following way: To the left of $\xi_{1}$ and to the right of $\xi_{N}$ we use a mesh of width $\Delta x$ that is aligned with $\xi_{1}$ respectively $\xi_{N}$. Inside each interval $D_{i}=\left(\xi_{i}, \xi_{i+1}\right)$ a mesh of the form $\left\{\xi_{i}+j \Delta x\right\}_{j=1}^{J}$ might not align with the point $\xi_{i+1}$. This happens precisely when $J \Delta x<\xi_{i+1}-\xi_{i}<(J+1) \Delta x$ in which case we set up the finer mesh $\left\{\xi_{i}+j \Delta x_{i}\right\}_{j=1}^{J+1}$ where $\Delta x_{i}=\frac{\xi_{i+1}-\xi_{i}}{J+1}$. Note that by definition we have

$$
\frac{1}{2} \Delta x \leq \Delta x_{i} \leq \Delta x
$$

In this way we can set up a spatial grid that is globally non-uniform, but uniform on each subdomain $D_{i}$. We want to point out the important fact that while the local grid sizes $\Delta x_{i}$ depend on the distance between neighboring discontinuities of $k$ (which we will assume to be random later) the upper and lower bounds of $\Delta x_{i}$ given by (5.1) are independ of $k$.

The resulting grid cells we denote by $\mathcal{C}_{j}=\left(x_{j-\frac{1}{2}}, x_{j+\frac{1}{2}}\right)$ for points $x_{j+\frac{1}{2}}$, such that for $j \in \mathbb{Z}$ we have $x_{j+\frac{1}{2}}-x_{j-\frac{1}{2}}=\Delta x_{i}$ for some $i=0, \ldots, N$. Here we used the notation $\Delta x_{0} \stackrel{\frac{1}{2}}{=} \Delta x_{N}=\Delta x$. The temporal grid cells we denote by $\mathcal{C}^{n}=\left[t^{n}, t^{n+1}\right)$ where $t^{n}=n \Delta t$ for $n=0, \ldots, M+1$. Since the grid is aligned with the discontinuities of $k$ we have $\xi_{i}=x_{P_{i}-\frac{1}{2}}$ for some integers $P_{i}, i=1, \ldots, N$.

We consider two-point numerical fluxes $F(u, v)$ that have the upwind property such that if $f^{\prime} \geq 0$ (which is the setting of the present paper), we have $F(u, v)=f(v)$. This includes the upwind flux, the Godunov flux, and the Engquist-Osher flux. The finite volume method we consider is the following [7]:

$$
\begin{gathered}
u_{j}^{0}=\frac{1}{\Delta x} \int_{\mathcal{C}_{j}} u_{0}(x) \mathrm{d} x, \quad j \in \mathbb{Z}, \\
u_{j}^{n+1}=u_{j}^{n}-\lambda_{i}\left(f^{(i)}\left(u_{j}^{n}\right)-f^{(i)}\left(u_{j-1}^{n}\right)\right), \quad n \geq 0, P_{i}<j<P_{i+1}, 0 \leq i \leq N, \\
u_{P_{i}}^{n+1}=\left(f^{(i)}\right)^{-1}\left(f^{(i-1)}\left(u_{P_{i}-1}^{n+1}\right)\right), \quad n \geq 0,0<i \leq N,
\end{gathered}
$$

where $P_{0}=-\infty, P_{N+1}=+\infty$, and $\lambda_{i}=\Delta t / \Delta x$. We assume that the grid discretization parameters satisfy the CFL condition

such that, in particular,

$$
\max _{i} \max _{u}\left(f^{(i)}\right)^{\prime}(u) \frac{\Delta t}{\Delta x} \leq \frac{1}{2}
$$

$$
\max _{i} \max _{u}\left(f^{(i)}\right)^{\prime}(u) \lambda_{i} \leq 1
$$

Note that the last line of (5.2) represents a discrete version of the Rankine-Hugoniot condition (3.2). Here, we use the ghost cells $\mathcal{C}_{P_{i}}, i=1, \ldots, N$ to explicitly enforce the Rankine-Hugoniot condition on the discrete level.

With the sequence of cell averages $\left(u_{j}^{n}\right)_{j, n}$ we associate the piecewise constant function $u_{\Delta x}(x, t)$ given by

$$
u_{\Delta x}(x, t)=u_{j}^{n}, \quad(x, t) \in \mathcal{C}_{j} \times \mathcal{C}^{n} .
$$

The following lemma shows that the finite volume method is stable in $\mathrm{L}^{\infty}$ and $\mathrm{L}^{1}$.

Lemma 5.1 (Stability of the finite volume method). If the numerical scheme (5.2) satisfies the CFL condition (5.3) we have the following stability estimates:

$$
\left\|u_{\Delta x}(\cdot, t)\right\|_{L^{\infty}(\mathbb{R})} \leq \frac{C_{f}}{\alpha}\left\|u_{0}\right\|_{L^{\infty}(\mathbb{R})}
$$


and

$$
\left\|u_{\Delta x}(\cdot, t)\right\|_{\mathrm{L}^{1}(\mathbb{R})} \leq\left\|u_{0}\right\|_{\mathrm{L}^{1}(\mathbb{R})}+C \operatorname{TV}\left(u_{0}\right) \Delta x .
$$

Proof. (1) We first prove the $\mathrm{L}^{\infty}$-bound. To that end, we show by induction over $i=0, \ldots, N$ that

$$
u_{j}^{n} \leq \max _{m=0, \ldots, i} \sup _{l=P_{m}, \ldots, P_{m+1}-1}\left(f^{(i)}\right)^{-1}\left(f^{(m)}\left(u_{l}^{0}\right)\right)
$$

for all $j=P_{i}, \ldots, P_{i+1}-1$ and $n=0, \ldots, M+1$. For $i=0$, standard techniques for finite volume methods for conservation laws show

$$
u_{j}^{n} \leq \max \left\{u_{j-1}^{n-1}, u_{j}^{n-1}\right\} \leq \ldots \leq \sup _{l<P_{1}} u_{l}^{0} .
$$

Assume now that (5.5) holds for some $i \in\{0, \ldots, N-1\}$ and all $j=P_{i}, \ldots, P_{i+1}-1$ and $n=0, \ldots, M+1$. Then we have for $j=P_{i+1}$

$$
u_{P_{i+1}}^{n}=\left(f^{(i+1)}\right)^{-1}\left(f^{(i)}\left(u_{P_{i+1}-1}^{n}\right)\right) \leq \max _{m=0, \ldots, i} \sup _{l=P_{m}, \ldots, P_{m+1}-1}\left(f^{(i+1)}\right)^{-1}\left(f^{(m)}\left(u_{l}^{0}\right)\right) .
$$

On the other hand, for $j \in\left\{P_{i+1}+1, \ldots, P_{i+2}-1\right\}$ we have as before

$$
u_{j}^{n} \leq \max \left\{u_{j-1}^{n-1}, \ldots, u_{j-1}^{1}, u_{j-1}^{0}, u_{j}^{0}\right\} \leq \ldots \leq \max \left\{u_{P_{i+1}}^{n-\left(j-P_{i+1}\right)}, \ldots, u_{P_{i+1}}^{1}, u_{P_{i+1}}^{0}, \ldots, u_{j}^{0}\right\} .
$$

By combining both estimates, we obtain for $j \in\left\{P_{i+1}, \ldots, P_{i+2}-1\right\}$

$$
\begin{aligned}
u_{j}^{n} & \leq \max \left\{\max _{l=P_{i+1}, \ldots, P_{i+2}-1} u_{l}^{0}, \max _{m=0, \ldots, i} \sup _{l=P_{m}, \ldots, P_{m+1}-1}\left(f^{(i+1)}\right)^{-1}\left(f^{(m)}\left(u_{l}^{0}\right)\right)\right\} \\
& =\max _{m=0, \ldots, i+1} \sup _{l=P_{m}, \ldots, P_{m+1}-1}\left(f^{(i+1)}\right)^{-1}\left(f^{(m)}\left(u_{l}^{0}\right)\right)
\end{aligned}
$$

which completes the induction. By taking absolute values in (5.5) we get for $j \in \mathbb{Z}$

$$
\left|u_{j}^{n}\right| \leq \frac{1}{\alpha} \max _{i=0, \ldots, N}\left\|f^{(i)}\right\|_{\text {Lip }}\left\|u_{0}\right\|_{L^{\infty}(\mathbb{R})} .
$$

Taking the supremum over $j$ yields the $\mathrm{L}^{\infty}$-bound (5.4).

(2) In order to prove the $\mathrm{L}^{1}$-bound note that we have the discrete entropy inequalities

$$
\left|u_{j}^{n+1}-c\right|-\left|u_{j}^{n}-c\right|+\lambda\left(q_{j}^{(i), n}-q_{j-1}^{(i), n}\right) \leq 0, \quad i=0, \ldots, N, j=P_{i}+1, \ldots, P_{i+1}-1
$$

for all $c \in \mathbb{R}$ (see [7]). Here, we have denoted $q_{j}^{(i), n}=\left|f^{(i)}\left(u_{j}^{n}\right)-f^{(i)}(c)\right|$. Taking $c=0$ and summing over $j \in \mathbb{Z} \backslash\left\{P_{1}, \ldots, P_{N}\right\}$ yields

$$
\sum_{j \neq P_{i}}\left|u_{j}^{n+1}\right| \leq \sum_{j \neq P_{i}}\left|u_{j}^{n}\right|-\lambda \sum_{i=0}^{N} \sum_{j=P_{i}+1}^{P_{i+1}-1}\left(q_{j}^{(i), n}-q_{j-1}^{(i), n}\right)=\sum_{j \neq P_{i}}\left|u_{j}^{n}\right| .
$$

Therefore, we have

$$
\sum_{j \in \mathbb{Z}}\left|u_{j}^{n+1}\right| \leq \sum_{j \in \mathbb{Z}}\left|u_{j}^{n}\right|+\sum_{i=1}^{N}\left(\left|u_{P_{i}}^{n+1}\right|-\left|u_{P_{i}}^{n}\right|\right) \leq \sum_{j \in \mathbb{Z}}\left|u_{j}^{n}\right|+\sum_{i=1}^{N} \frac{1}{\alpha}\left\|f^{(i-1)}\right\|_{\text {Lip }}\left|u_{P_{i}-1}^{n+1}-u_{P_{i}-1}^{n}\right|
$$


and hence

$$
\sum_{j \in \mathbb{Z}}\left|u_{j}^{n+1}\right| \leq \sum_{j \in \mathbb{Z}}\left|u_{j}^{0}\right|+\sum_{i=0}^{N} \frac{1}{\alpha}\left\|f^{(i-1)}\right\|_{\text {Lip }} \sum_{m=0}^{n}\left|u_{P_{i}-1}^{m+1}-u_{P_{i}-1}^{m}\right| .
$$

In Lemma 4.6 of [7], it was shown that for all $i=0, \ldots, N$ we have

$$
\sum_{m=0}^{n}\left|u_{P_{i}-1}^{m+1}-u_{P_{i}-1}^{m}\right| \leq C \operatorname{TV}\left(u_{0}\right)
$$

which together with the foregoing estimate finally yields

$$
\left\|u_{\Delta x}(\cdot, t)\right\|_{L^{1}(\mathbb{R})} \leq\left\|u_{0}\right\|_{L^{1}(\mathbb{R})}+C \operatorname{TV}\left(u_{0}\right) \Delta x .
$$

In order to prove error estimates of the Monte Carlo and multilevel Monte Carlo finite volume method we will need the following convergence rate estimate which was proved in [7].

Theorem 5.2 (Convergence rate of the finite volume method [7]). Let $f, k$, and $u_{0}$ satisfy Assumption 3.1 and the discretization parameters satisfy the CFL condition (5.3). Then the finite volume approximation $u_{\Delta x}$ given by the scheme (5.2) converges towards the unique entropy solution u of (4.1) almost everywhere and in $\mathrm{L}^{1}(\mathbb{R} \times(0, T))$. In particular, we have the following convergence rate estimate

$$
\left\|u(\cdot, t)-u_{\Delta x}(\cdot, t)\right\|_{L^{1}(\mathbb{R})} \leq C \Delta x^{\frac{1}{2}}
$$

for all $0 \leq t \leq T$.

Note that the convergence rate estimate (5.6) is optimal in the sense that the exponent $\frac{1}{2}$ cannot be improved without further assumptions on the initial datum [7] (see [47] for an overview of the literature regarding optimal convergence rates of finite volume methods for conservation laws without spatial dependency).

Remark 5.3. We want to point out that the constant $C$ in (5.6) depends only on $\operatorname{TV}\left(u_{0}\right),\left\|u_{0}\right\|_{L^{\infty}},\|f\|_{\infty}, \alpha$ and the number of discontinuities of $k$. In particular, for random data given according to Definition 4.1 all those quantities are uniformly bounded and thus for random entropy solutions the constant $C$ in (5.6) is integrable in $\omega$.

Remark 5.4. Reasoning as for entropy solutions, the finite volume approximation satisfies

$$
\left\|u_{\Delta x}(\cdot, t)\right\|_{\mathrm{L}^{p}(D)} \leq|D|^{\frac{1}{p}}\left\|u_{\Delta x}(\cdot, t)\right\|_{\mathrm{L}^{\infty}(D)} \leq C\left\|u_{0}\right\|_{\mathrm{L}^{\infty}(D)}
$$

for all $1 \leq p \leq \infty$. Like in Lemma 4.5, this translates into the following probabilistic bound:

$$
\left\|u_{\Delta x}(\cdot, t)\right\|_{\mathrm{L}^{r}\left(\Omega ; \mathrm{L}^{p}(D)\right)} \leq C\left\|u_{0}\right\|_{\mathrm{L}^{r}\left(\Omega ; \mathrm{L}^{\infty}(D)\right)}
$$

for all $0 \leq t \leq T$ and $1 \leq p \leq \infty$.

For the rest of this paper, we will consider entropy solutions on a bounded interval $D \subset \mathbb{R}$ with periodic boundary conditions. With the usual arguments, all previous results concerning entropy solutions and their finite volume approximations carry over to this setting verbatim. Note that restricting ourselves to a bounded domain will enable us to prove error estimates of the Monte Carlo and multilevel Monte Carlo finite volume method also in $\mathrm{L}^{2}\left(\Omega ; \mathrm{L}^{1}(D)\right)$ (cf. [45]). 


\subsection{Monte Carlo finite volume method}

We now consider the random conservation law with discontinuous flux (4.1) and introduce and analyze the Monte Carlo finite volume method.

Given $M \in \mathbb{N}$, we generate $M$ independent and identically distributed samples $\left(\hat{f}^{i}, \hat{k}^{i}, \hat{u}_{0}^{i}\right)_{i=1}^{M}$ of given random data $\left(u_{0}, k, f\right)$. Let now $\hat{u}_{\Delta x}^{i}(\cdot, t), i=1, \ldots, M$, denote the numerical solutions generated by the finite volume method (5.2) at time $t$ corresponding to the sample $\left(\hat{f}^{i}, \hat{k}^{i}, \hat{u}_{0}^{i}\right)$. Then, the $M$-sample MCFVM approximation to $\mathbb{E}[u(\cdot, t)]$ is defined as

$$
E_{M}\left[u_{\Delta x}(\cdot, t)\right]=\frac{1}{M} \sum_{i=1}^{M} \hat{u}_{\Delta x}^{i}(\cdot, t) .
$$

As mentioned earlier the approximation error of the MCFVM has a component coming from the statistical sampling error and one from the deterministic discretization error. We will make this statement precise in the following theorem.

Theorem 5.5 (MCFVM error estimate). Let $\left(u_{0}, k, f\right)$ be random data and $u$ the corresponding random entropy solution of (4.1). Assume that $u_{0}$ satisfies the $r$-th moment condition

$$
\left\|u_{0}\right\|_{L^{r}\left(\Omega ; L^{\infty}(D)\right)}<\infty
$$

for some $1<r \leq \infty$. Assume further that we are given a FVM (5.2) such that the CFL condition (5.3) holds. Then, for each $1 \leq p \leq \infty$ and $0 \leq t \leq T$ and for $q=\min (2, r)>1$, the $M C F V M$ approximation satisfies the error estimate

$$
\left\|\mathbb{E}[u(\cdot, t)]-E_{M}\left[u_{\Delta x}(\cdot, t)\right]\right\|_{L^{q}\left(\Omega ; L^{p}(D)\right)} \leq C\left(M^{\frac{1-q}{q}}\left\|u_{0}\right\|_{L^{r}\left(\Omega ; L^{\infty}(D)\right)}+\left\|u_{0}\right\|_{L^{r}\left(\Omega ; L^{\infty}(D)\right)}^{1-\frac{1}{p}} \Delta x^{\frac{1}{2 p}}\right) .
$$

In particular, the MCFVM approximation converges towards $\mathbb{E}[u(\cdot, t)]$ in $\mathrm{L}^{q}\left(\Omega ; \mathrm{L}^{p}(D)\right)$ as $M \rightarrow \infty$ and $\Delta x \rightarrow 0$.

Proof. We use the triangle inequality to get

$$
\begin{aligned}
& \left\|\mathbb{E}[u(\cdot, t)]-E_{M}\left[u_{\Delta x}(\cdot, t)\right]\right\|_{L^{q}\left(\Omega ; \mathrm{L}^{p}(D)\right)} \\
& \quad \leq\left\|\mathbb{E}[u(\cdot, t)]-E_{M}[u(\cdot, t)]\right\|_{L^{q}\left(\Omega ; \mathrm{L}^{p}(D)\right)}+\left\|E_{M}[u(\cdot, t)]-E_{M}\left[u_{\Delta x}(\cdot, t)\right]\right\|_{\mathrm{L}^{q}\left(\Omega ; \mathrm{L}^{p}(D)\right)}
\end{aligned}
$$

and estimate the resulting two terms separately. For the first term in (5.9), we distinguish the two cases $p \geq q$ and $p<q$.

(1) We first consider the case $p \geq q$. According to Lemma 4.5 we have

$$
\|u(\cdot, t)\|_{\mathrm{L}^{r}\left(\Omega ; \mathrm{L}^{p}(D)\right)} \leq C\left\|u_{0}\right\|_{\mathrm{L}^{r}\left(\Omega ; \mathrm{L}^{\infty}(D)\right)}
$$

and thus $u(\cdot, t) \in \mathrm{L}^{r}\left(\Omega ; \mathrm{L}^{p}(D)\right)$. Therefore, we can apply Theorem 2.5 to get

$$
\begin{aligned}
\left\|\mathbb{E}[u(\cdot, t)]-E_{M}[u(\cdot, t)]\right\|_{\mathrm{L}^{q}\left(\Omega ; \mathrm{L}^{p}(D)\right)} & \leq C M^{\frac{1-q}{q}}\|u(\cdot, t)\|_{\mathrm{L}^{q}\left(\Omega ; \mathrm{L}^{p}(D)\right)} \\
& \leq C M^{\frac{1-q}{q}}\|u(\cdot, t)\|_{\mathrm{L}^{r}\left(\Omega ; \mathrm{L}^{p}(D)\right)} \\
& \leq C M^{\frac{1-q}{q}}\left\|u_{0}\right\|_{\mathrm{L}^{r}\left(\Omega ; \mathrm{L}^{\infty}(D)\right)} .
\end{aligned}
$$

(2) In the case $p<q$, we can apply Hölder's inequality to estimate

$$
\left\|\mathbb{E}[u(\cdot, t)]-E_{M}[u(\cdot, t)]\right\|_{L^{q}\left(\Omega ; \mathrm{L}^{p}(D)\right)} \leq C\left\|\mathbb{E}[u(\cdot, t)]-E_{M}[u(\cdot, t)]\right\|_{L^{q}\left(\Omega ; L^{q}(D)\right)} .
$$


Again, we want to employ Theorem 2.5. To that end, we note that because of Lemma 4.5 and the fact that $q \leq r$ we have

$$
\|u(\cdot, t)\|_{\mathrm{L}^{q}\left(\Omega ; \mathrm{L}^{q}(D)\right)} \leq C\left\|u_{0}\right\|_{\mathrm{L}^{q}\left(\Omega ; \mathrm{L}^{\infty}(D)\right)} \leq C\left\|u_{0}\right\|_{\mathrm{L}^{r}\left(\Omega ; \mathrm{L}^{\infty}(D)\right)}
$$

and therefore $u(\cdot, t) \in \mathrm{L}^{q}\left(\Omega ; \mathrm{L}^{q}(D)\right)$ and we can apply Theorem 2.5 to get

$$
\left\|\mathbb{E}[u(\cdot, t)]-E_{M}[u(\cdot, t)]\right\|_{L^{q}\left(\Omega ; L^{q}(D)\right)} \leq C M^{\frac{1-q}{q}}\|u(\cdot, t)\|_{L^{q}\left(\Omega ; L^{q}(D)\right)} \leq C M^{\frac{1-q}{q}}\left\|u_{0}\right\|_{L^{r}\left(\Omega ; L^{\infty}(D)\right)} .
$$

Hence, for all $1 \leq p \leq \infty$, we get

$$
\left\|\mathbb{E}[u(\cdot, t)]-E_{M}[u(\cdot, t)]\right\|_{L^{q}\left(\Omega ; \mathrm{L}^{p}(D)\right)} \leq C M^{\frac{1-q}{q}}\left\|u_{0}\right\|_{\mathrm{L}^{r}\left(\Omega ; \mathrm{L}^{\infty}(D)\right)} .
$$

On the other hand, for the second term in (5.9) we can use the triangle inequality and the linearity of the expected value to obtain

$$
\begin{aligned}
\left\|E_{M}[u(\cdot, t)]-E_{M}\left[u_{\Delta x}(\cdot, t)\right]\right\|_{L^{q}\left(\Omega ; \mathrm{L}^{p}(D)\right)} & \leq \frac{1}{M} \sum_{i=1}^{M}\left\|\hat{u}^{i}(\cdot, t)-\hat{u}_{\Delta x}^{i}(\cdot, t)\right\|_{\mathrm{L}^{q}\left(\Omega ; \mathrm{L}^{p}(D)\right)} \\
& =\left\|u(\cdot, t)-u_{\Delta x}(\cdot, t)\right\|_{\mathrm{L}^{q}\left(\Omega ; \mathrm{L}^{p}(D)\right)} .
\end{aligned}
$$

Using the interpolation inequality between $\mathrm{L}^{1}$ and $\mathrm{L}^{\infty}$, the $\mathrm{L}^{\infty}$-bound for both $u(\cdot, t)$ and $u_{\Delta x}(\cdot, t)($ see $(3.3)$ respectively (5.4)), and the convergence rate estimate (5.6), we get

$$
\begin{aligned}
\left\|u(\cdot, t)-u_{\Delta x}(\cdot, t)\right\|_{\mathrm{L}^{q}\left(\Omega ; \mathrm{L}^{p}(D)\right)} & \leq\left\|u(\cdot, t)-u_{\Delta x}(\cdot, t)\right\|_{\mathrm{L}^{q}\left(\Omega ; \mathrm{L}^{1}(D)\right)}^{\frac{1}{p}}\left\|u(\cdot, t)-u_{\Delta x}(\cdot, t)\right\|_{\mathrm{L}^{q}\left(\Omega ; \mathrm{L}^{\infty}(D)\right)}^{1-\frac{1}{p}} \\
& \leq C\left\|u_{0}\right\|_{\mathrm{L}^{r}\left(\Omega ; \mathrm{L}^{\infty}(D)\right)}^{1-\frac{1}{p}} \Delta x^{\frac{1}{2 p}},
\end{aligned}
$$

which completes the proof.

Note that the computations in the proof of the error estimate (5.8) are also valid if $r=1$ (and thus $q=1$ ). However, in that case the right-hand side does not decrease as $M \rightarrow \infty$.

\subsection{Multilevel Monte Carlo finite volume method}

Instead of just considering Monte Carlo samples of a single fixed resolution of the finite volume method, we now detail the corresponding multilevel variant - the multilevel Monte Carlo finite volume method. The idea of MLMC discretization of differential equations with random parameters was proposed by Giles in $[18,19]$ based upon earlier work by Heinrich on numerical quadrature [24]. The key ingredient of the MLMCFVM is simultaneous MC sampling on different levels of resolution of the finite volume method with level-dependent numbers $M_{l}$ of MC samples.

To that end, we generate a sequence of finite volume approximations $U(\cdot, t):=\left(u_{l}(\cdot, t)\right)_{l=0}^{L}$ on grids with cell sizes $\Delta x_{l}$ and time steps $\Delta t_{l}$ (subject to the CFL condition $\left.(5.3)\right)$ and set $u_{\Delta x_{-1}}(\cdot, t)=0$. Then, we have

$$
\mathbb{E}\left[u_{\Delta x_{L}}(\cdot, t)\right]=\mathbb{E}\left[\sum_{l=0}^{L}\left(u_{\Delta x_{l}}(\cdot, t)-u_{\Delta x_{l-1}}(\cdot, t)\right)\right]=\sum_{l=0}^{L} \mathbb{E}\left[u_{\Delta x_{l}}(\cdot, t)-u_{\Delta x_{l-1}}(\cdot, t)\right] .
$$

We now approximate each term $\mathbb{E}\left[u_{\Delta x_{l}}(\cdot, t)-u_{\Delta x_{l-1}}(\cdot, t)\right]$ by a Monte Carlo estimator with $M_{l}$ samples. The resulting MLMCFVM approximation to $\mathbb{E}[u(\cdot, t)]$ then is

$$
E^{L}[U(\cdot, t)]=\sum_{l=0}^{L} E_{M_{l}}\left[u_{\Delta x_{l}}(\cdot, t)-u_{\Delta x_{l-1}}(\cdot, t)\right]
$$


In the following convergence analysis, we will assume for simplicity that $\Delta x_{l}=2^{-l} \Delta x_{0}, l=0, \ldots, L$, for some $\Delta x_{0}>0$.

As for the MCFVM, we want to obtain a rate at which $E^{L}[U(\cdot, t)]$ converges towards $\mathbb{E}[u(\cdot, t)]$ in terms of the number of MC samples $M_{l}$ and the spatial resolution $\Delta x_{l}$ on each level $l=0, \ldots, L$.

Theorem 5.6 (MLMCFVM error estimate). Let $L>0,\left(u_{0}, k, f\right)$ be random data, and $u$ the corresponding random entropy solution of (4.1). Assume that $u_{0}$ satisfies

$$
\left\|u_{0}\right\|_{\mathrm{L}^{r}\left(\Omega ; \mathrm{L}^{\infty}(D)\right)}<\infty
$$

for some $1<r \leq \infty$. Assume further that we are given a FVM (5.2) such that the CFL condition (5.3) holds. Then, for each $0 \leq t \leq T$, for any sequence $\left(M_{l}\right)_{l=0}^{L}$ of sample sizes at mesh level $l$ the MLMCFVM approximation (5.10) satisfies the following error estimate for $q=\min (2, r)>1$

$$
\begin{aligned}
\| \mathbb{E}[u(\cdot, t)]- & E^{L}[U(\cdot, t)] \|_{L^{q}\left(\Omega ; L^{p}(\mathbb{R})\right)} \\
& \leq C\left(\left\|u_{0}\right\|_{L^{1}\left(\Omega ; L^{\infty}(D)\right)}^{1-\frac{1}{p}} \Delta x_{L}^{\frac{1}{2 p}}+\left\|u_{0}\right\|_{L^{q}\left(\Omega ; L^{\infty}(D)\right)} M_{0}^{\frac{1-q}{q}}+\left\|u_{0}\right\|_{L^{q}\left(\Omega ; L^{\infty}(D)\right)}^{1-\frac{1}{p}} \sum_{l=0}^{L} M_{l}^{\frac{1-q}{q}} \Delta x_{l}^{\frac{1}{2 \widetilde{p}}}\right)
\end{aligned}
$$

where $\widetilde{p}=\max (p, q)$. In particular, for fixed $L$ the $M L M C F V M$ approximation $E^{L}[U(\cdot, t)]$ converges towards $\mathbb{E}[u(\cdot, t)]$ in $\mathrm{L}^{q}\left(\Omega ; \mathrm{L}^{p}(D)\right)$ as $M_{l} \rightarrow \infty$ and $\Delta x_{0} \rightarrow 0$.

Proof. Using the triangle inequality and the linearity of the expectation, we get

$$
\begin{aligned}
\| \mathbb{E}[u(\cdot, t)]- & E^{L}[U(\cdot, t)] \|_{\mathrm{L}^{q}\left(\Omega ; \mathrm{L}^{p}(D)\right)} \\
\leq & \left\|\mathbb{E}[u(\cdot, t)]-\mathbb{E}\left[u_{\Delta x_{L}}(\cdot, t)\right]\right\|_{\mathrm{L}^{q}\left(\Omega ; \mathrm{L}^{p}(D)\right)}+\left\|\mathbb{E}\left[u_{\Delta x_{L}}(\cdot, t)\right]-E^{L}[U(\cdot, t)]\right\|_{\mathrm{L}^{q}\left(\Omega ; \mathrm{L}^{p}(D)\right)} \\
= & \left\|\mathbb{E}\left[u(\cdot, t)-u_{\Delta x_{L}}(\cdot, t)\right]\right\|_{\mathrm{L}^{q}\left(\Omega ; \mathrm{L}^{p}(D)\right)} \\
& +\left\|\sum_{l=0}^{L}\left(\mathbb{E}\left[u_{\Delta x_{l}}(\cdot, t)-u_{\Delta x_{l-1}}(\cdot, t)\right]-E_{M_{l}}\left[u_{\Delta x_{l}}(\cdot, t)-u_{\Delta x_{l-1}}(\cdot, t)\right]\right)\right\|_{\mathrm{L}^{q}\left(\Omega ; \mathrm{L}^{p}(D)\right)} \\
\leq & \left\|\mathbb{E}\left[u(\cdot, t)-u_{\Delta x_{L}}(\cdot, t)\right]\right\|_{\mathrm{L}^{q}\left(\Omega ; \mathrm{L}^{p}(D)\right)} \\
& +\sum_{l=0}^{L}\left\|\mathbb{E}\left[u_{\Delta x_{l}}(\cdot, t)-u_{\Delta x_{l-1}}(\cdot, t)\right]-E_{M_{l}}\left[u_{\Delta x_{l}}(\cdot, t)-u_{\Delta x_{l-1}}(\cdot, t)\right]\right\|_{\mathrm{L}^{q}\left(\Omega ; \mathrm{L}^{p}(D)\right)} .
\end{aligned}
$$

For the first term, note that the function $\mathbb{E}\left[u(\cdot, t)-u_{\Delta x_{L}}(\cdot, t)\right]$ is deterministic and thus we can use the convergence rate estimate (5.6) to get

$$
\begin{aligned}
\| \mathbb{E}[u(\cdot, t) & \left.-u_{\Delta x_{L}}(\cdot, t)\right] \|_{\mathrm{L}^{q}\left(\Omega ; \mathrm{L}^{p}(D)\right)} \\
& \leq\left\|u(\cdot, t)-u_{\Delta x_{L}}(\cdot, t)\right\|_{\mathrm{L}^{1}\left(\Omega ; \mathrm{L}^{p}(D)\right)}^{\frac{1}{p}} \\
& \leq\left\|u(\cdot, t)-u_{\Delta x_{L}}(\cdot, t)\right\|_{\mathrm{L}^{1}\left(\Omega ; \mathrm{L}^{1}(D)\right)}^{\frac{1}{p}}\left\|u(\cdot, t)-u_{\Delta x_{L}}(\cdot, t)\right\|_{\mathrm{L}^{1}\left(\Omega ; \mathrm{L}^{\infty}(D)\right)}^{1-\frac{1}{p}} \\
& \leq C\left\|u_{0}\right\|_{\mathrm{L}^{1}\left(\Omega ; \mathrm{L}^{\infty}(D)\right)}^{1-\frac{1}{2 p}} \Delta x_{L}^{\frac{1}{2 p}} .
\end{aligned}
$$

We now estimate the summands in the second term. Similarly to the proof of Theorem 5.5 we distinguish the two cases $p \geq q$ and $p<q$.

(1) We first consider the case $p \geq q$. Because of the triangle inequality and (5.7) we have

$$
\left\|u_{\Delta x_{l}}(\cdot, t)-u_{\Delta x_{l-1}}(\cdot, t)\right\|_{L^{r}\left(\Omega ; L^{p}(D)\right)} \leq C\left\|u_{0}\right\|_{L^{r}\left(\Omega ; L^{\infty}(D)\right)}
$$


and thus $u_{\Delta x_{l}}(\cdot, t)-u_{\Delta x_{l-1}}(\cdot, t) \in \mathrm{L}^{r}\left(\Omega ; \mathrm{L}^{p}(D)\right)$. Therefore we can apply Theorem 2.5 to get

$$
\begin{aligned}
\left\|\mathbb{E}\left[u_{\Delta x_{l}}(\cdot, t)-u_{\Delta x_{l-1}}(\cdot, t)\right]-E_{M_{l}}\left[u_{\Delta x_{l}}(\cdot, t)-u_{\Delta x_{l-1}}(\cdot, t)\right]\right\|_{L^{q}\left(\Omega ; \mathrm{L}^{p}(D)\right)} \\
\leq C M_{l}^{\frac{1-q}{q}}\left\|u_{\Delta x_{l}}(\cdot, t)-u_{\Delta x_{l-1}}(\cdot, t)\right\|_{L^{q}\left(\Omega ; \mathrm{L}^{p}(D)\right)} .
\end{aligned}
$$

(2) In the case $p<q$, we can apply Hölder's inequality to estimate

$$
\begin{aligned}
\| \mathbb{E}\left[u_{\Delta x_{l}}(\cdot, t)-u_{\Delta x_{l-1}}(\cdot, t)\right] & -E_{M_{l}}\left[u_{\Delta x_{l}}(\cdot, t)-u_{\Delta x_{l-1}}(\cdot, t)\right] \|_{L^{q}\left(\Omega ; L^{p}(D)\right)} \\
& \leq C\left\|\mathbb{E}\left[u_{\Delta x_{l}}(\cdot, t)-u_{\Delta x_{l-1}}(\cdot, t)\right]-E_{M_{l}}\left[u_{\Delta x_{l}}(\cdot, t)-u_{\Delta x_{l-1}}(\cdot, t)\right]\right\|_{L^{q}\left(\Omega ; \mathrm{L}^{q}(D)\right)} .
\end{aligned}
$$

Following the same steps as in case (2) in the proof of Theorem 5.5 for $u_{\Delta x_{l}}(\cdot, t)-u_{\Delta x_{l-1}}(\cdot, t)$ instead of $u(\cdot, t)$ and using (5.7) instead of Lemma 4.5, we see that $u_{\Delta x_{l}}(\cdot, t)-u_{\Delta x_{l-1}}(\cdot, t) \in \mathrm{L}^{q}\left(\Omega ; \mathrm{L}^{q}(D)\right)$. Thus, we can apply Theorem 2.5 again and get

$$
\begin{aligned}
&\left\|\mathbb{E}\left[u_{\Delta x_{l}}(\cdot, t)-u_{\Delta x_{l-1}}(\cdot, t)\right]-E_{M_{l}}\left[u_{\Delta x_{l}}(\cdot, t)-u_{\Delta x_{l-1}}(\cdot, t)\right]\right\|_{L^{q}\left(\Omega ; \mathrm{L}^{q}(D)\right)} \\
& \leq C M_{l}^{\frac{1-q}{q}}\left\|u_{\Delta x_{l}}(\cdot, t)-u_{\Delta x_{l-1}}(\cdot, t)\right\|_{L^{q}\left(\Omega ; \mathrm{L}^{q}(D)\right)} .
\end{aligned}
$$

Combining both cases, we get

$$
\begin{aligned}
\left\|\mathbb{E}\left[u_{\Delta x_{l}}(\cdot, t)-u_{\Delta x_{l-1}}(\cdot, t)\right]-E_{M_{l}}\left[u_{\Delta x_{l}}(\cdot, t)-u_{\Delta x_{l-1}}(\cdot, t)\right]\right\|_{L^{q}\left(\Omega ; \mathrm{L}^{p}(D)\right)} & \\
& \leq C M_{l}^{\frac{1-q}{q}}\left\|u_{\Delta x_{l}}(\cdot, t)-u_{\Delta x_{l-1}}(\cdot, t)\right\|_{L^{q}\left(\Omega ; \mathrm{L}^{\tilde{p}}(D)\right)}
\end{aligned}
$$

where $\widetilde{p}=\max (p, q)$. Now, we can use the triangle inequality to get

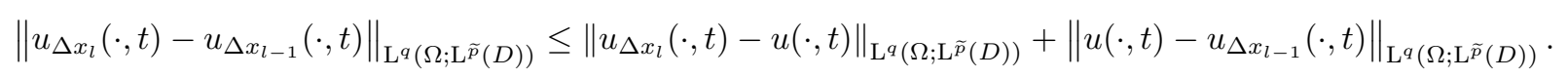

For $l>0$, we can use the interpolation inequality between $\mathrm{L}^{1}$ and $\mathrm{L}^{\infty}$, the $\mathrm{L}^{1}$ and $\mathrm{L}^{\infty}$ bounds of the entropy solution and finite volume approximations (see (3.3) respectively (5.4)), and the convergence rate estimate (5.6) to get

$$
\begin{aligned}
\| u_{\Delta x_{l}}(\cdot, t)- & u(\cdot, t)\left\|_{\mathrm{L}^{q}\left(\Omega ; \mathrm{L}^{\tilde{p}}(D)\right)}+\right\| u(\cdot, t)-u_{\Delta x_{l-1}}(\cdot, t) \|_{\mathrm{L}^{q}\left(\Omega ; \mathrm{L}^{\tilde{p}}(D)\right)} \\
\leq & \left\|u_{\Delta x_{l}}(\cdot, t)-u(\cdot, t)\right\|_{\mathrm{L}^{q}\left(\Omega ; \mathrm{L}^{1}(D)\right)}^{\frac{1}{\tilde{p}}}\left\|u_{\Delta x_{l}}(\cdot, t)-u(\cdot, t)\right\|_{\mathrm{L}^{q}\left(\Omega ; \mathrm{L}^{\infty}(D)\right)}^{1-\frac{1}{\tilde{p}}} \\
& +\left\|u(\cdot, t)-u_{\Delta x_{l-1}}(\cdot, t)\right\|_{\mathrm{L}^{q}\left(\Omega ; \mathrm{L}^{1}(D)\right)}^{\frac{1}{\tilde{p}}}\left\|u(\cdot, t)-u_{\Delta x_{l-1}}(\cdot, t)\right\|_{\mathrm{L}^{q}\left(\Omega ; \mathrm{L}^{\infty}(D)\right)}^{1-\frac{1}{p}} \\
\leq & C\left\|u_{0}\right\|_{\mathrm{L}^{q}\left(\Omega ; \mathrm{L}^{\infty}(D)\right)}^{1-\frac{1}{\tilde{p}}}\left(\Delta x_{l}^{\frac{1}{2 \widetilde{p}}}+\Delta x_{l-1}^{\frac{1}{2 \widetilde{p}}}\right) \\
\leq & C\left\|u_{0}\right\|_{\mathrm{L}^{q}\left(\Omega ; \mathrm{L}^{\infty}(D)\right)}^{1-\frac{1}{\tilde{p}}} \Delta x_{l}^{\frac{1}{2 \widetilde{p}}}
\end{aligned}
$$

Similarly, for $l=0$ (note that $u_{\Delta x_{-1}}=0$ ), the convergence rate estimate (5.6) and the bound from Lemma 4.5 give

$$
\begin{aligned}
\left\|u_{\Delta x_{0}}(\cdot, t)\right\|_{\mathrm{L}^{q}\left(\Omega ; \mathrm{L}^{\widetilde{p}}(D)\right)} & \leq\left\|u_{\Delta x_{0}}(\cdot, t)-u(\cdot, t)\right\|_{\mathrm{L}^{q}\left(\Omega ; \mathrm{L}^{\widetilde{p}}(D)\right)}+\|u(\cdot, t)\|_{\mathrm{L}^{q}\left(\Omega ; \mathrm{L}^{\widetilde{p}}(D)\right)} \\
& \leq C\left(\left\|u_{0}\right\|_{\mathrm{L}^{q}\left(\Omega ; \mathrm{L}^{\infty}(D)\right)}^{1-\frac{1}{\tilde{p}}} \Delta x_{0}^{\frac{1}{2 \widetilde{p}}}+\left\|u_{0}\right\|_{\mathrm{L}^{q}\left(\Omega ; \mathrm{L}^{\infty}(D)\right)}\right)
\end{aligned}
$$


Combining all estimates finally gives

$$
\begin{aligned}
& \left\|\mathbb{E}[u(\cdot, t)]-E^{L}[U(\cdot, t)]\right\|_{L^{q}\left(\Omega ; \mathrm{L}^{p}(\mathbb{R})\right)} \\
& \quad \leq C\left(\left\|u_{0}\right\|_{\mathrm{L}^{1}\left(\Omega ; \mathrm{L}^{\infty}(D)\right)}^{1-\frac{1}{p}} \Delta x_{L}^{\frac{1}{2 p}}+\left\|u_{0}\right\|_{\mathrm{L}^{q}\left(\Omega ; \mathrm{L}^{\infty}(D)\right)} M_{0}^{\frac{1-q}{q}}+\left\|u_{0}\right\|_{\mathrm{L}^{q}\left(\Omega ; \mathrm{L}^{\infty}(D)\right)}^{1-\frac{1}{p}} \sum_{l=0}^{L} M_{l}^{\frac{1-q}{q}} \Delta x_{l}^{\frac{1}{2 \tilde{p}}}\right) .
\end{aligned}
$$

\subsection{Work estimates and sample number optimization}

In order to analyze the efficiency of the MC and MLMCFVM, it is important to estimate the computational work which is needed to compute one approximation of the solution by the deterministic FVM and how it increases with respect to mesh refinement. Here, by computational work, we understand the number of floating point operations performed when executing an algorithm and we assume that this in turn is proportional to the runtime of the algorithm.

In practice, we deal with bounded domains instead of working on the whole real line and thus the number of grid cells scales as $1 / \Delta x$. For the deterministic FVM (5.2) the number of floating point operations per time step is proportional to the number of cells in the spatial domain, hence the computational work can be bounded by $C \Delta t^{-1} \Delta x^{-1}$. Considering the CFL condition (5.3), we thus obtain the computational work estimate

$$
W^{\mathrm{FVM}}(\Delta x) \leq C \Delta x^{-2}
$$

for the deterministic FVM approximation. However, for the sake of generality, we will in the following only assume that the computational work scales as

$$
W^{\mathrm{FVM}}(\Delta x) \leq C \Delta x^{-w}
$$

for some $w>0$. As seen before, we have the $\mathrm{L}^{p}$ convergence rate estimate

$$
\left\|u(\cdot, t)-u_{\Delta x}(\cdot, t)\right\|_{L^{p}(D)} \leq C \Delta x^{\frac{s}{p}}
$$

(for $s=\frac{1}{2}$ ) which yields the following deterministic convergence rate with respect to work:

$$
\left\|u(\cdot, t)-u_{\Delta x}(\cdot, t)\right\|_{\mathrm{L}^{p}(D)} \leq C\left(W^{\mathrm{FVM}}\right)^{-\frac{s}{w p}} .
$$

In particular, for $p=1, w=2$, and $s=\frac{1}{2}$ we have

$$
\left\|u(\cdot, t)-u_{\Delta x}(\cdot, t)\right\|_{\mathrm{L}^{1}(D)} \leq C\left(W^{\mathrm{FVM}}\right)^{-\frac{1}{4}} .
$$

\subsubsection{Work estimates for the MCFVM approximation}

Since for the Monte Carlo finite volume method $M$ deterministic finite volume approximations need to be computed, each of which require work as in (5.12), the computational work for the MCFVM is bounded as

$$
W_{M}^{\mathrm{MC}} \leq C M \Delta x^{-w} .
$$

In order to obtain the order of convergence of the approximation error in terms of computational work, we equilibrate the terms $M^{\frac{1-q}{q}}$ and $\Delta x^{\frac{s}{p}}$ in (5.8) by choosing $M=C \Delta x^{\frac{s q}{p(1-q)}}$. Inserting this into the work bound (5.14) yields

$$
W_{M}^{\mathrm{MCFVM}} \leq C \Delta x^{\frac{s q-w p(1-q)}{p(1-q)}}
$$


such that we obtain from (5.8)

$$
\left\|\mathbb{E}[u(\cdot, t)]-E_{M}\left[u_{\Delta x}(\cdot, t)\right]\right\|_{L^{q}\left(\Omega ; \mathrm{L}^{p}(D)\right)} \leq C \Delta x^{\frac{s}{p}} \leq C\left(W_{M}^{\mathrm{MC}}\right)^{-\frac{s}{w p+s \frac{q}{q-1}}} .
$$

Note that, since $q /(q-1)$ is positive, we have

$$
\frac{s}{w p+s \frac{q}{q-1}} \leq \frac{s}{w p}
$$

and thus the rate (5.15) is worse than the error rate in terms of computational work (5.13) of the deterministic finite volume method.

In particular, for $p=1$ and $r \geq 2$ (which implies $q=2$ ), and taking into account that $w=2$ and $s=\frac{1}{2}$, the rate (5.15) reads

$$
\left\|\mathbb{E}[u(\cdot, t)]-E_{M}\left[u_{\Delta x}(\cdot, t)\right]\right\|_{\mathrm{L}^{2}\left(\Omega ; \mathrm{L}^{1}(D)\right)} \leq C\left(W_{M}^{\mathrm{MC}}\right)^{-\frac{1}{6}} .
$$

\subsubsection{Optimal sample numbers for the MLMCFVM approximation}

In [32], Koley et al. showed the following general result for multilevel Monte Carlo finite volume methods which we can apply to our case to determine the number of samples needed at each level $l$ such that, given an error tolerance $\varepsilon>0$, the computational work of the MLMCFVM is minimal.

Lemma 5.7 ([32], Lem. 4.9). Assume that the work of a multilevel Monte Carlo finite volume method with $L$ discretization levels scales asymptotically as

$$
W_{L}^{\mathrm{MLMC}}=C \sum_{l=0}^{L} M_{l} \Delta x_{l}^{-w}
$$

for some $w>0$ and that the approximation error (raised to the $q$-th power) scales as

$$
\operatorname{Err}_{L}=C\left(\Delta x_{L}^{\frac{s q}{p}}+M_{0}^{1-q}+\sum_{l=0}^{L} M_{l}^{1-q} \Delta x_{l}^{\frac{s q}{p}}\right)
$$

where $\widetilde{p}=\max (p, q)$ (cf. (5.11)). Then, given an error tolerance $\varepsilon>0$, the optimal sample numbers $M_{l}$ minimizing the computational work given the error tolerance $\varepsilon$ are given by

$$
M_{0} \simeq\left(\frac{1+\Delta x_{0}^{\frac{s}{\tilde{p}}} \sum_{l=1}^{L} 2^{l\left(w \frac{q-1}{q}-\frac{s}{\tilde{p}}\right)}}{\varepsilon-\Delta x_{L}^{\frac{s q}{p}}}\right)^{\frac{1}{q-1}}
$$

and

$$
M_{l} \simeq M_{0} \Delta x_{0}^{\frac{s}{p}} 2^{-l\left(\frac{s}{\tilde{p}}+\frac{w}{q}\right)}, \quad \text { for } l>0,
$$

where $\simeq$ indicates that this is the number of samples up to a constant which is independent of $l$ and $L$. The minimal amount of work then is

$$
W_{L}^{\mathrm{MLMC}} \simeq \Delta x_{0}^{-w}\left(1+\Delta x_{0}^{\frac{s}{\hat{p}}} \sum_{l=1}^{L} 2^{l\left(w \frac{q-1}{q}-\frac{s}{\tilde{p}}\right)}\right)\left(\frac{1+\Delta x_{0}^{\frac{s}{p}} \sum_{l=1}^{L} 2^{l\left(w \frac{q-1}{q}-\frac{s}{\tilde{p}}\right)}}{\varepsilon-\Delta x_{0}^{\frac{s q}{p}} 2^{-L \frac{q s}{p}}}\right)^{\frac{1}{q-1}} .
$$

Lemma 5.7 can be used to derive a rate for the approximation error of the MLMCFVM in terms of the computational work. 
Corollary 5.8. In addition to the assumptions of Lemma 5.7, assume that $w \frac{q-1}{q}-\frac{s}{\tilde{p}}>0$ and that $L$ and $\Delta x_{0}$ are large enough such that

$$
\Delta x_{L}^{\frac{s}{p} \frac{q}{q-1}-w}>\Delta x_{0}^{-w}
$$

where $\widetilde{p}=\max (p, q)$ and $w$ is as in (5.7). Then, for each $0 \leq t \leq T$ and for $q=\min (2, r)$ the $\mathrm{L}^{q}\left(\Omega ; \mathrm{L}^{p}(D)\right)$ approximation error of the MLMCFVM (5.10) scales with respect to computational work as

$$
\left\|\mathbb{E}[u(\cdot, t)]-E^{L}[U(\cdot, t)]\right\|_{L^{q}\left(\Omega ; \mathrm{L}^{p}(D)\right)} \leq C\left(W_{L}^{\mathrm{MLMC}}\right)^{-\frac{s}{w p+s \frac{\hat{p}-p}{\tilde{p}} \frac{q}{q-1}}} .
$$

Proof. Since $\left(w \frac{q-1}{q}-\frac{s}{\widetilde{p}}\right)>0$ the sums in the expression for $W_{M}^{\mathrm{MLMC}}$ from Lemma 5.7 are dominated by $2^{L\left(w \frac{q-1}{q}-\frac{s}{p}\right)}$. Choosing $\varepsilon=2 \Delta x_{L}^{\frac{s q}{p}}$ and using that $\Delta x_{L}^{\frac{s}{p} \frac{q}{q-1}-w}>\Delta x_{0}^{-w}$ in the last step, we find

$$
\begin{aligned}
W_{L}^{\mathrm{MLMC}} & \simeq \Delta x_{0}^{-w}\left(1+\Delta x_{0}^{\frac{s}{\tilde{p}}} 2^{L\left(w \frac{q-1}{q}-\frac{s}{\tilde{p}}\right)}\right)\left(\frac{1+\Delta x_{0}^{\frac{s}{p}} 2^{L\left(w \frac{q-1}{q}-\frac{s}{\tilde{p}}\right)}}{\Delta x_{L}^{\frac{s q}{p}}}\right)^{\frac{1}{q-1}} \\
& \simeq \Delta x_{0}^{-w} \Delta x_{L}^{-\frac{s q}{p(q-1)}}\left(1+\Delta x_{0}^{\frac{s}{p}} 2^{L\left(w \frac{q-1}{q}-\frac{s}{\tilde{p}}\right)}\right)^{\frac{q}{q-1}} \\
& \simeq \Delta x_{L}^{-\frac{s q}{p(q-1)}}\left(\Delta x_{0}^{-w}+\Delta x_{L}^{\frac{s}{p} \frac{q}{q-1}-w}\right) \\
& \simeq \Delta x_{L}^{s\left(\frac{1}{\tilde{p}}-\frac{1}{p}\right) \frac{q}{q-1}-w} .
\end{aligned}
$$

Thus, we have

$$
\left\|\mathbb{E}[u(\cdot, t)]-E^{L}[U(\cdot, t)]\right\|_{L^{2}\left(\Omega ; L^{1}(D)\right)}=\varepsilon^{\frac{1}{q}} \simeq \Delta x_{L}^{\frac{s}{p}} \simeq\left(W_{L}^{\mathrm{MLMC}}\right)^{-\frac{s}{w p+s \frac{\hat{p}-p}{\tilde{p}} \frac{q}{q-1}}} .
$$

Since $\frac{(\widetilde{p}-p)}{\widetilde{p}}$ and $\frac{q}{(q-1)}$ are nonnegative, we have

$$
\frac{s}{w p+s \frac{\widetilde{p}-p}{\tilde{p}} \frac{q}{q-1}} \leq \frac{s}{w p}
$$

and thus the error rate in terms of the computational work (5.18) of the MLMCFVM is worse than the error rate (5.13) for the deterministic scheme. However, since $\frac{\widetilde{p}-p}{\widetilde{p}} \leq 1-\frac{p}{q} \leq 1$, we have

$$
\frac{s}{w p+s \frac{\widetilde{p}-p}{\widetilde{p}} \frac{q}{q-1}} \geq \frac{s}{w p+s \frac{q}{q-1}}
$$

and thus the error rate (5.18) of the MLMCFVM constitutes an improvement over the (single-level) MCFVM, cf. (5.15).

Note that, in particular, for $p=1$ and $r \geq 2$ (which implies $q=2$ and $\widetilde{p}=2$ ), and taking into account that $w=2$ and $s=\frac{1}{2}$, the error rate (5.18) reads

$$
\left\|\mathbb{E}[u(\cdot, t)]-E^{L}[U(\cdot, t)]\right\|_{L^{2}\left(\Omega ; \mathrm{L}^{1}(D)\right)} \leq C\left(W_{L}^{\mathrm{MLMC}}\right)^{-\frac{1}{5}}
$$




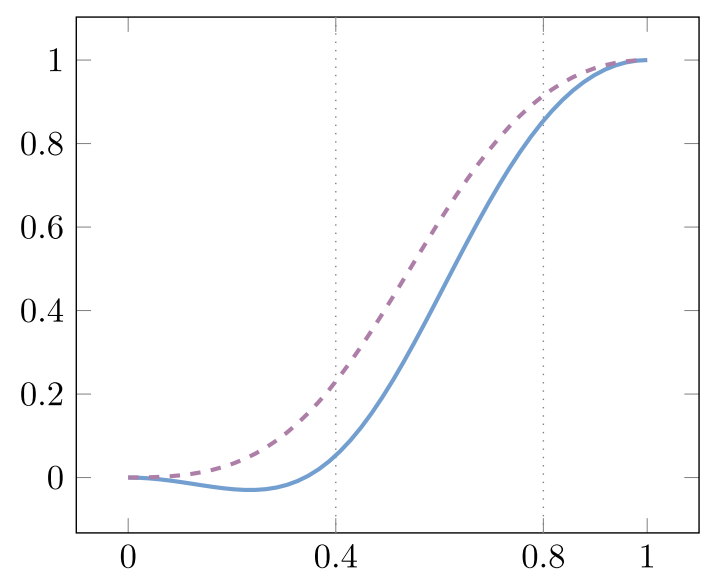

Figure 1. Two possible fluxes of the form (6.1) for $k(x)=0.7$ (dashed line) and $k(x)=2.3$ (solid line)

\section{NUMERICAL EXPERIMENTS}

In this section, we present numerical experiments motivated by two-phase flow in a heterogeneous porous medium $^{1}$. The time evolution of the oil saturation $u \in[0,1]$ can be modeled by $(1.1)$ where the flux is given by

$$
f(k(x), u)=\frac{\lambda_{\mathrm{o}}(u)}{\lambda_{\mathrm{o}}(u)+\lambda_{\mathrm{w}}(u)}\left(1-k(x) \lambda_{\mathrm{w}}(u)\right),
$$

see Example 8.2 of [25]. Here, the functions $\lambda_{\mathrm{o}}$ and $\lambda_{\mathrm{w}}$ denote the phase mobilities/relative permeabilities of the oil and the water phase, respectively. Typically, one uses the simple expressions

$$
\lambda_{\mathrm{o}}(u)=u^{2}, \quad \lambda_{\mathrm{w}}(u)=(1-u)^{2}
$$

which we will also do in the first two subsequent experiments. The coefficient $k$ in (6.1) corresponds to the absolute permeability of the medium. Since the medium is usually layered to some extent throughout the reservoir and even continuously varying geology is typically mapped onto some grid, the coefficient $k$ is often modeled as a piecewise constant function [23].

Since numerical experiments for conservation laws where the initial datum or the flux is uncertain have been reported in other works (albeit without spatially discontinuous flux), we will here focus on numerical experiments where, in particular, the discontinuous coefficient $k$ is subject to randomness. We consider the initial datum

$$
u_{0}(x)= \begin{cases}0.8, & -0.9<x<-0.2, \\ 0.4, & \text { otherwise },\end{cases}
$$

on the spatial domain $D=[-1,1]$ with periodic boundary conditions. Figure 1 shows two examples of fluxes of the form (6.1) and indicates the relevant domain determined by the initial datum (6.2). In all experiments we use $\lambda=\frac{\Delta t}{\Delta x}=0.2$ in the finite volume approximation (5.2).

When choosing the number of samples for the MLMC estimator we use the formulae (5.16) and (5.17) with "=" replacing " $\simeq$ " and rounding to the next biggest integer. Here we use $p=1, r=q=2, w=2, s=\frac{1}{2}$, and $\varepsilon=2 \Delta x_{L}^{2 s}$ in (5.16) and $(5.17)^{2}$.

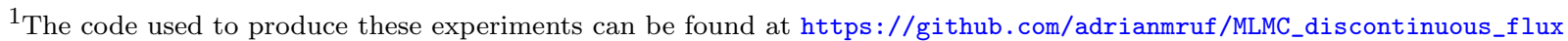

${ }^{2}$ For example, for $L=7$ and $\Delta x_{0}=2^{-4}$ we use $\left(M_{l}\right)_{l=0}^{L}=(95646,20107,8454,3555,1495,629,265,112)$ samples.
} 
In order to compute an estimate of the approximation error

$$
\left\|\mathbb{E}[u(\cdot, T)]-E^{L}[U(\cdot, T)]\right\|_{L^{2}\left(\Omega ; L^{1}(D)\right)}=\left(\mathbb{E}\left[\left\|\mathbb{E}[u(\cdot, T)]-E^{L}[U(\cdot, T)]\right\|_{L^{1}(D)}^{2}\right]\right)^{\frac{1}{2}}
$$

we use the root mean square estimator introduced in [38]: We denote by $U_{\text {ref }}(\cdot, T)$ a reference solution and by $\left(U_{i}(\cdot, T)\right)_{i=1}^{K}$ a sequence of independent approximate solutions $E^{L}[U(\cdot, T)]$ obtained by running the MLMCFVM estimator with $L$ levels $K$ times. Then, we estimate the relative error by

$$
\mathcal{R M S}=\left(\frac{1}{K} \sum_{i=1}^{K}\left(\mathcal{R M S}_{i}\right)^{2}\right)^{\frac{1}{2}}
$$

where

$$
\mathcal{R M S}_{i}=100 \times \frac{\left\|U_{\text {ref }}(\cdot, T)-U_{i}(\cdot, T)\right\|_{\mathrm{L}^{1}(D)}}{\left\|U_{\text {ref }}(\cdot, T)\right\|_{\mathrm{L}^{1}(D)}} .
$$

Here, as suggested in [38], we use $K=30$ which was shown to be sufficient for most problems. In each experiment, as a reference approximation $U_{\text {ref }}(\cdot, T)$ of $\mathbb{E}[u(\cdot, T)]$, we use a solution computed by the MLMCFVM with $\Delta x_{0}=2^{-4}$ and $L=8$ which entails using $2^{13}$ cells on the finest level.

In our figures we also indicate the approximated standard deviation. To that end, we approximate the variance by

$$
V_{L}=\sum_{l=0}^{L} E_{M_{l}}\left[\left(u_{\Delta x_{l}}(\cdot, T)-u_{\Delta x_{l-1}}(\cdot, T)-E_{M_{l}}\left[u_{\Delta x_{l}}(\cdot, T)-u_{\Delta x_{l-1}}(\cdot, T)\right]\right)^{2}\right] .
$$

\subsection{Uncertain position of rock layer interface}

For our first numerical experiment we will model the absolute permeability parameter as

$$
k(x)= \begin{cases}1, & x<\sigma(\omega), \\ 2, & x>\sigma(\omega)\end{cases}
$$

corresponding to an uncertain position of the interface between two rock types in the reservoir. Here, the random variable $\sigma$ is uniformly distributed in $[-0.3,0.3]$. Figure 2a shows two samples of the approximate random entropy solution (with $\sigma=-0.3$ and $\sigma=0.3$, respectively) calculated using $2^{10}$ grid points at time $T=0.2$ and Figure $2 \mathrm{~b}$ shows an estimate of the expectation $\mathbb{E}[u(\cdot, T)]$ computed by the MLMCFVM with $\Delta x_{0}=2^{-4}$ and $L=7$.

Table 1 and Figure 3 show the estimated $\mathcal{R} \mathcal{M S}$ error as a function of the number of levels. In particular, Table 1a shows the observed order of convergence (OOC) with respect to $\Delta x_{L}$ while Table $1 \mathrm{~b}$ shows the observed order of convergence with respect to the computational work calculated based on a best linear fit under the assumptions that $\mathcal{R M S} \sim\left(\Delta x_{L}\right)^{r_{1}}$ and $\mathcal{R} \mathcal{M S} \sim(\text { work })^{r_{2}}$. Here, we use the runtime as a surrogate for the computational work. We observe that in Experiment 1 both rates are better than the rates guaranteed by our convergence analysis.

\subsection{Uncertain absolute permeabilities}

For our second numerical experiment we will model the absolute permeability parameter as

$$
k(x)= \begin{cases}\xi_{1}(\omega), & x<0, \\ \xi_{2}(\omega), & x>0\end{cases}
$$

corresponding to uncertain absolute permeabilities of two rock layers. Here, the random variables $\xi_{1}$ and $\xi_{2}$ are independent and uniformly distributed in $[0.7,1.3]$ and $[1.7,2.3]$, respectively. 


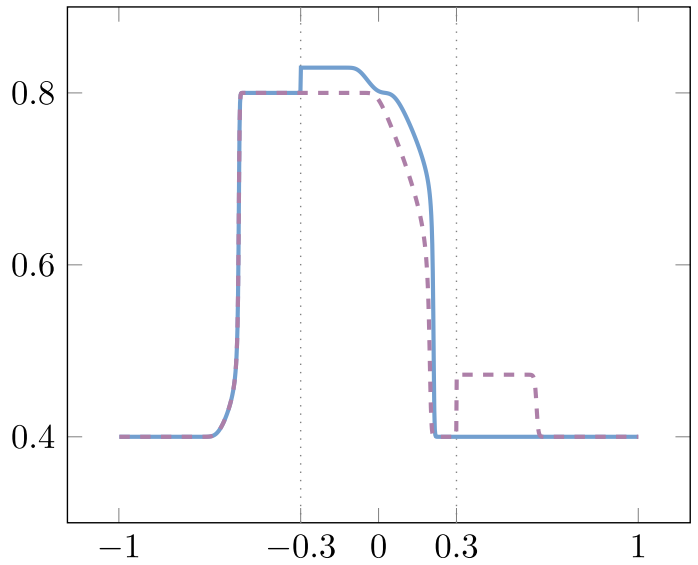

(A)

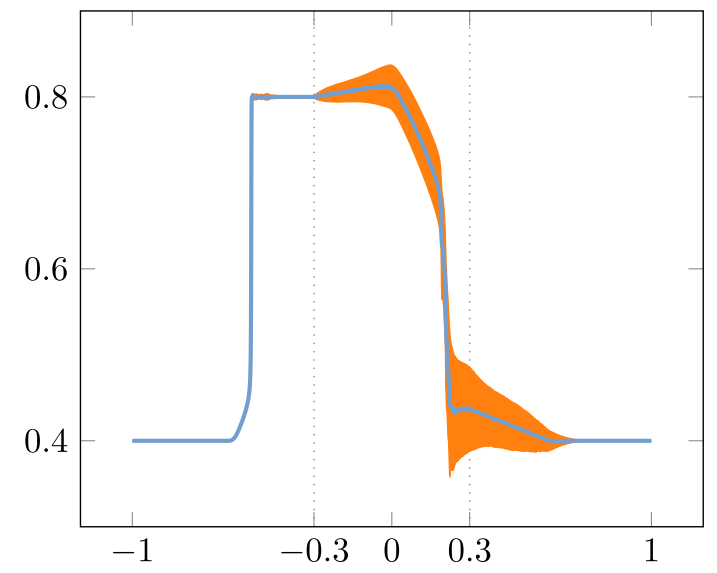

(B)

FiguRE 2. Two samples and a MLMCFVM approximation of the (mean of the) random entropy solution for Experiment 1 with $T=0.2$ and $\lambda=0.2$. The orange area indicates the area between the mean \pm standard deviation. For each sample the discontinuity of $k$ is located in the interval between the dotted lines. (A) Two samples of the random entropy solution $(\sigma=-0.3$ (solid line), $\sigma=0.3$ (dashed line), $\left.\Delta x=2^{-9}\right)$. (B) MLMCFVM approximation $\left(\Delta x_{0}=2^{-4}, L=7\right.$ ).

TABLE 1. $\mathcal{R} \mathcal{M S}$ error in Experiment 1 as a function of the finest grid resolution $\Delta x_{L}$ and as a function of the work (here measured by the runtime in s) for various values of $L$ and for $\Delta x_{0}=2^{-4}$.

(a) $\mathcal{R M S}$ versus $\Delta x_{L}$

\begin{tabular}{llll}
\hline \hline$L$ & $\Delta x_{L}$ & $\mathcal{R M S}$ & OOC \\
\hline 1 & $2^{-5}$ & 4.03 & \\
2 & $2^{-6}$ & 2.53 & \\
3 & $2^{-7}$ & 1.53 & \\
4 & $2^{-8}$ & 0.88 & \\
5 & $2^{-9}$ & 0.49 & \\
6 & $2^{-10}$ & 0.24 & 0.80 \\
\hline
\end{tabular}

(b) $\mathcal{R M S}$ versus work.

\begin{tabular}{llll}
\hline \hline$L$ & Runtime & $\mathcal{R M S}$ & OOC \\
\hline 1 & 0.09 & 4.03 & \\
2 & 0.23 & 2.53 & \\
3 & 0.73 & 1.53 & \\
4 & 2.65 & 0.88 & \\
5 & 10.12 & 0.49 & \\
6 & 39.23 & 0.24 & -0.45 \\
\hline
\end{tabular}

Figure 4a shows two samples of the approximate random entropy solution (with $\left(\xi_{1}, \xi_{2}\right)=(1.3,1.7)$ and $\left(\xi_{1}, \xi_{2}\right)=(0.7,2.3)$, respectively) calculated using $2^{10}$ grid points at time $T=0.2$ and Figure $4 \mathrm{~b}$ shows an estimate of the expectation $\mathbb{E}[u(\cdot, T)]$ computed by the MLMCFVM with $\Delta x_{0}=2^{-4}$ and $L=7$.

Table 2 and Figure 5 again show the root mean square error estimate and the observed order of convergence with respect to $\Delta x_{L}$ and with respect to the computational work. As before, we observe that the observed convergence rates are better than the theoretical bounds.

\subsection{Uncertain position of rock layer interface and absolute and relative permeabilities}

In our last numerical experiment we will model the absolute permeability parameter as

$$
k(x)= \begin{cases}\xi_{1}(\omega), & x<\sigma(\omega), \\ \xi_{2}(\omega), & x>\sigma(\omega)\end{cases}
$$




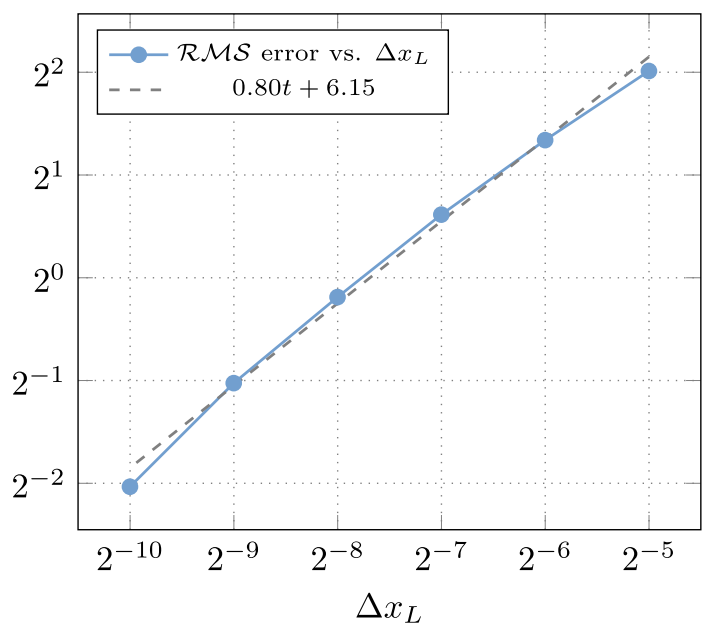

(A)

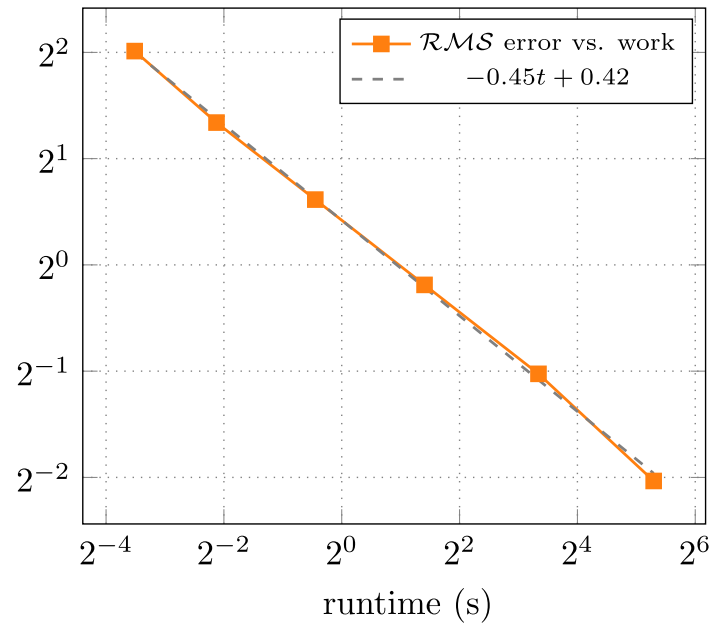

(B)

FiguRE 3. $\mathcal{R} \mathcal{M S}$ error in Experiment 1 as a function of the finest grid resolution $\Delta x_{L}$ and as a function of the work (here measured by the runtime in $s$ ) corresponding to the values in Table 1 . The dashed lines indicate the observed order of convergence based on a best linear fit. (A) $\mathcal{R M S}$ error versus $\Delta x_{L}$. (B) $\mathcal{R} \mathcal{M S}$ error versus work.

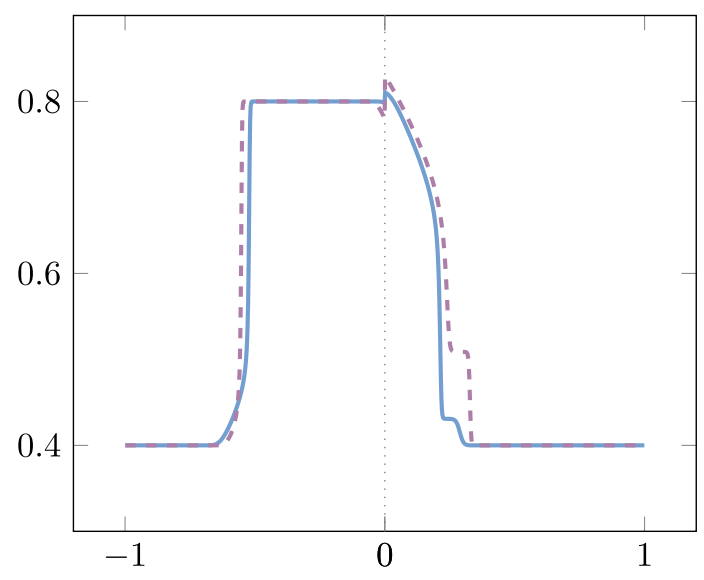

(A)

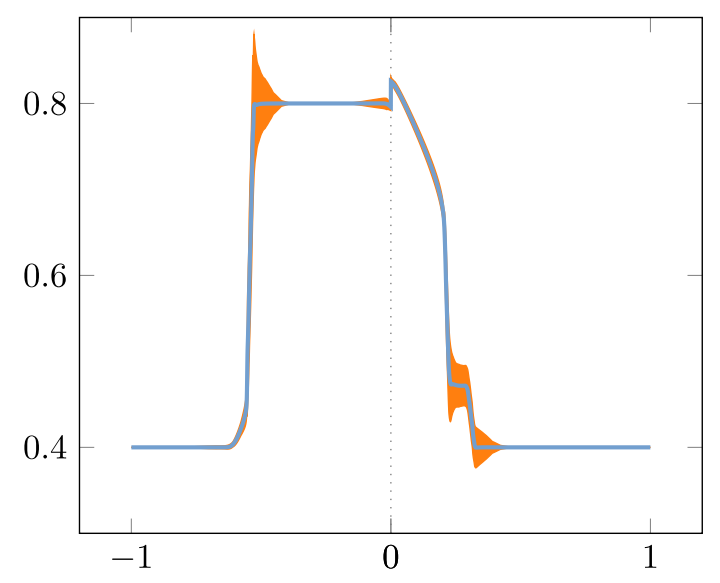

(B)

FIgURE 4. Two samples and a MLMCFVM approximation of the (mean of the) random entropy solution for Experiment 2 with $T=0.2$ and $\lambda=0.2$. The orange area indicates the area between the mean \pm standard deviation and the dotted line marks the (fixed) position of the discontinuity of $k$. (A) Two samples of the random entropy solution $\left(\left(\xi_{1}, \xi_{2}\right)=(1.3,1.7)\right.$ (solid line), $\left(\xi_{1}, \xi_{2}\right)=(0.7,2.3)$ (dashed line), $\left.\Delta x=2^{-9}\right)$. (B) MLMCFVM approximation $\left(\Delta x_{0}=2^{-4}, L=7\right)$. 
TABLE 2. $\mathcal{R M S}$ error in Experiment 2 as a function of the finest grid resolution $\Delta x_{L}$ and as a function of the work (here measured by the runtime in $\mathrm{s}$ ) for various values of $L$ and for $\Delta x_{0}=2^{-4}$.

(a) $\mathcal{R M S}$ versus $\Delta x_{L}$.

\begin{tabular}{llll}
\hline \hline$L$ & $\Delta x_{L}$ & $\mathcal{R M S}$ & OOC \\
\hline 1 & $2^{-5}$ & 3.86 & \\
2 & $2^{-6}$ & 2.32 & \\
3 & $2^{-7}$ & 1.41 & \\
4 & $2^{-8}$ & 0.82 & \\
5 & $2^{-9}$ & 0.45 & \\
6 & $2^{-10}$ & 0.22 & 0.82 \\
\hline
\end{tabular}

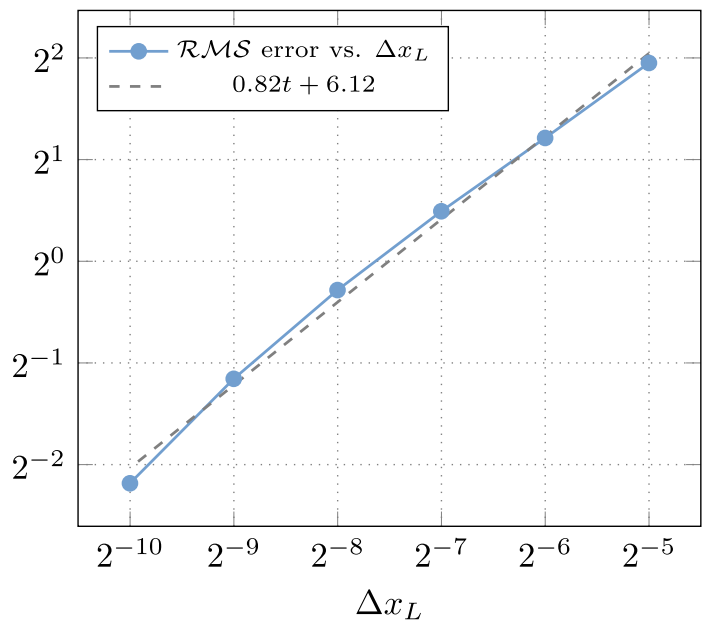

(A) (b) $\mathcal{R M S}$ versus work.

\begin{tabular}{llll}
\hline \hline$L$ & Runtime $(\mathrm{s})$ & $\mathcal{R M S}$ & OOC \\
\hline 1 & 0.07 & 3.86 & \\
2 & 0.18 & 2.32 & \\
3 & 0.64 & 1.41 & \\
4 & 2.52 & 0.82 & \\
5 & 9.88 & 0.45 & \\
6 & 38.16 & 0.22 & -0.44 \\
\hline
\end{tabular}

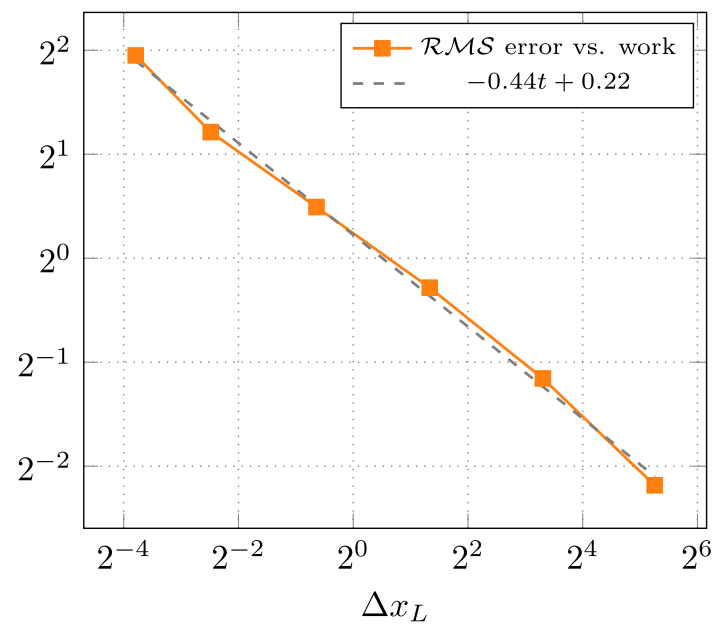

(B)

FiguRE 5. $\mathcal{R} \mathcal{M S}$ error in Experiment 2 as a function of the finest grid solution $\Delta x_{L}$ and as a function of the work (here measured by the runtime in $s$ ) corresponding to the values in Table 2. The dotted lines indicate the observed order of convergence based on a best linear fit.

(A) $\mathcal{R} \mathcal{M S}$ error versus $\Delta x_{L}$. (B) $\mathcal{R} \mathcal{M S}$ error versus work.

corresponding to an uncertain position of the interface between two rock types as well as uncertain absolute permeabilities of the rock layers. Here, the random variables $\xi_{1}, \xi_{2}$, and $\sigma$ are uniformly distributed in $[0.7,1.3]$, $[1.7,2.3]$ and $[-0.3,0.3]$, respectively. Furthermore, we will model the relative permeabilities $\lambda_{o}$ and $\lambda_{w}$ in $(6.1)$ as

$$
\lambda_{o}(u)=u^{p(\omega)}, \quad \lambda_{w}(u)=(1-u)^{p(\omega)}
$$

where the random exponent $p$ is uniformly distributed in [1.5, 2.5]. Here, $\xi_{1}, \xi_{2}, \sigma$ and $p$ are mutually independent.

Figure 6a shows two samples of the approximate random entropy solution (with $\left(\xi_{1}, \xi_{2}, \sigma, p\right)=$ $(0.3,-0.3,-0.3,1.5)$ and $\left(\xi_{1}, \xi_{2}, \sigma, p\right)=(-0.3,0.3,0.3,2.5)$, respectively) calculated using $2^{10}$ grid points at time $T=0.2$ and Figure $6 \mathrm{~b}$ shows an estimate of the expectation $\mathbb{E}[u(\cdot, T)]$ computed by the MLMCFVM with $\Delta x_{0}=2^{-4}$ and $L=7$. 


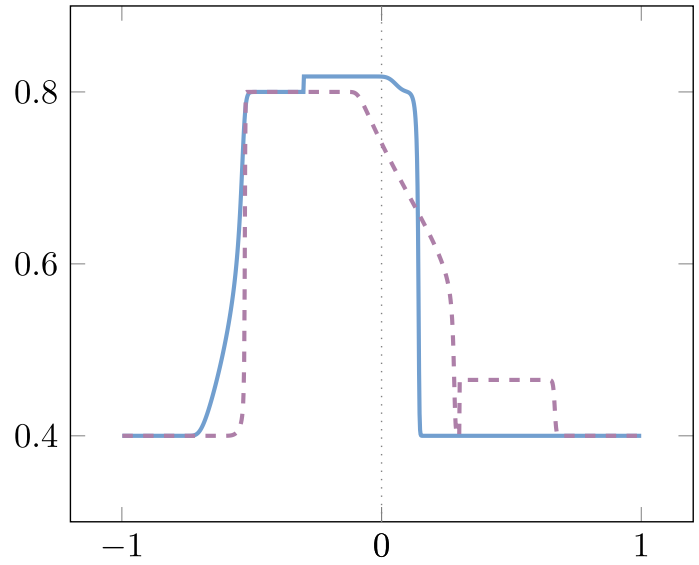

(A)

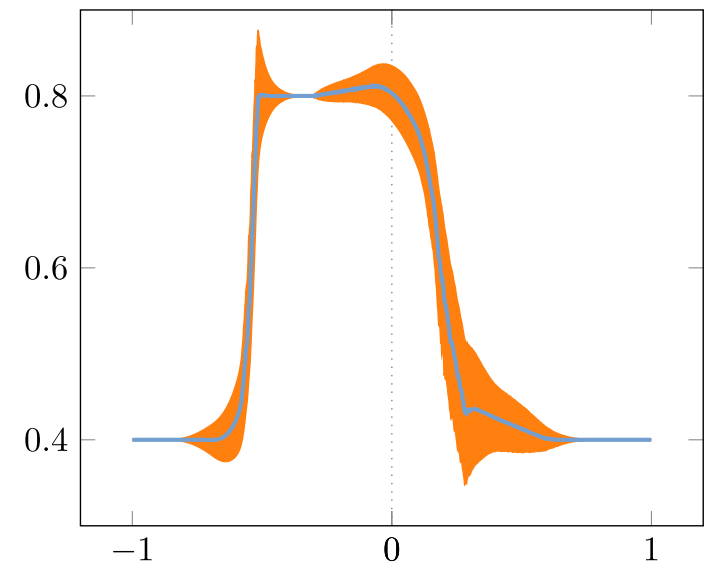

(B)

Figure 6 . Two samples and a MLMCFVM approximation of the (mean of the) random entropy solution for Experiment 3 with $T=0.2$ and $\lambda=0.2$. The orange area indicates the area between the mean \pm standard deviation and the dotted line marks the (fixed) position of the discontinuity of $k$. (A) Two samples of the random entropy solution $\left(\left(\xi_{1}, \xi_{2}, \sigma, p\right)=(1.3,1.7,-0.3,1.5)\right.$ (solid line), $\left(\xi_{1}, \xi_{2}, \sigma, p\right)=(0.7,2.3,0.3,2.5)$ (dashed line), $\left.\Delta x=2^{-9}\right)$. (B) MLMCFVM approximation $\left(\Delta x_{0}=2^{-4}, L=7\right)$.

TABLE 3. $\mathcal{R} \mathcal{M S}$ error in Experiment 3 as a function of the finest grid resolution $\Delta x_{L}$ and as a function of the work (here measured by the runtime in s) for various values of $L$ and for $\Delta x_{0}=2^{-4}$.

(a) $\mathcal{R M S}$ versus $\Delta x_{L}$.

\begin{tabular}{llll}
\hline \hline$L$ & $\Delta x_{L}$ & $\mathcal{R M S}$ & OOC \\
\hline & & & \\
1 & $2^{-5}$ & 3.44 & \\
2 & $2^{-6}$ & 1.97 & \\
3 & $2^{-7}$ & 1.14 & \\
4 & $2^{-8}$ & 0.63 & \\
5 & $2^{-9}$ & 0.33 & \\
6 & $2^{-10}$ & 0.17 & 0.86 \\
\hline
\end{tabular}

(b) $\mathcal{R M S}$ versus work.

\begin{tabular}{llll}
\hline \hline$L$ & Runtime (s) & $\mathcal{R M S}$ & OOC \\
\hline & & & \\
1 & 0.08 & 3.44 & \\
2 & 0.21 & 1.97 & \\
3 & 0.74 & 1.14 & \\
4 & 3.07 & 0.63 & \\
5 & 12.93 & 0.33 & \\
6 & 55.93 & 0.17 & -0.45 \\
\hline
\end{tabular}

Table 3 and Figure 7 again show the root mean square error estimate and the observed order of convergence with respect to $\Delta x_{L}$ and with respect to the computational work. Notably, the observed convergence rates are very similar to those in Experiments 1 and 2 despite the four dimensional parameter space.

\section{Conclusion}

In this paper, we have considered conservation laws with discontinuous flux where the model parameters, i.e., the initial datum, the flux function, and the discontinuous spatial dependency coefficient, are uncertain. Based on adapted entropy solutions for the deterministic case, we have introduced a notion of random entropy solutions and have proved well-posedness.

To numerically approximate the mean of a random entropy solution, we have proposed Monte Carlo methods coupled with a class of finite volume methods suited for conservation laws with discontinuous flux. Our 


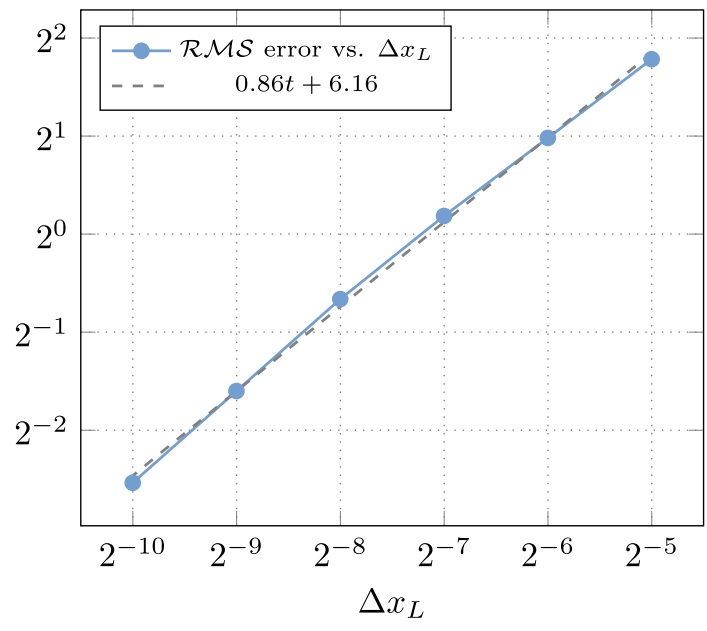

(A)

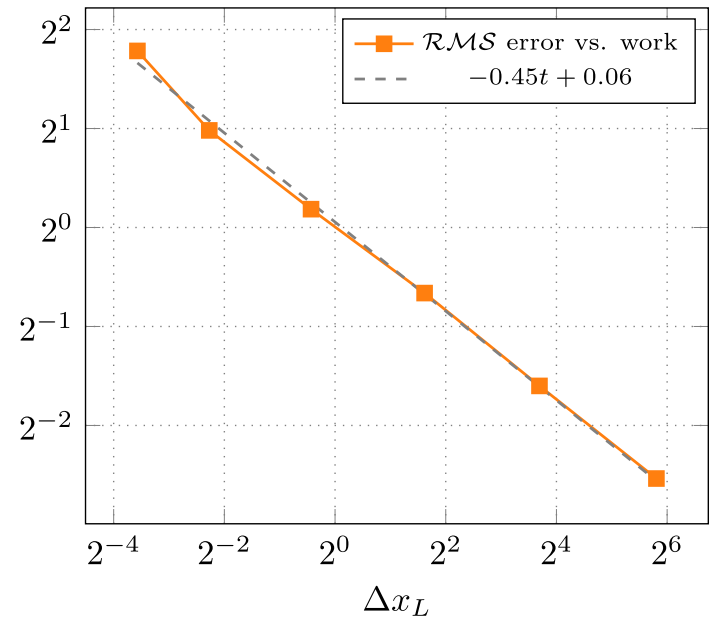

(B)

FiguRE 7. $\mathcal{R M S}$ error in Experiment 3 as a function of the finest grid solution $\Delta x_{L}$ and as a function of the work (here measured by the runtime in $s$ ) corresponding to the values in Table 3 . The dotted lines indicate the observed order of convergence based on a best linear fit. (A) $\mathcal{R} \mathcal{M S}$ error versus $\Delta x_{L}$. (B) $\mathcal{R} \mathcal{M S}$ error versus work.

convergence analysis includes convergence rate estimates for the Monte Carlo and multilevel Monte Carlo finite volume method. Further, we have provided error versus work rates which show that the multilevel Monte Carlo finite volume method is more efficient than the (single-level) Monte Carlo finite volume method.

We have presented numerical experiments motivated by two-phase flow in heterogeneous porous media, e.g., oil reservoirs with different rock layers. The numerical experiments verify our theoretical results concerning convergence rates of the multilevel Monte Carlo finite volume method.

As a possible direction of future research, we want to mention that - from a practical standpoint - it would be desirable to design multilevel Monte Carlo finite volume methods based on finite volume methods that require no processing of the flux discontinuities. Such numerical methods have been considered in [15, 51], however, there are currently no convergence rate results available for these methods.

Acknowledgements. The work of JB was supported by German Priority Programme 1648 (SPPEXA) and the ModCompShock EU Project. The work of NHR was performed while visiting the University of Würzburg supported by the Giovanni-Prodi Chair Position. JB, CK, and NHR were supported by DAAD (German Academic Exchange Service) and the Research Council of Norway.

\section{REFERENCES}

[1] R. Aae Klausen and N.H. Risebro, Stability of conservation laws with discontinuous coefficients. J. Differ. Equ. 157 (1999) $41-60$.

[2] R. Abgrall, A simple, flexible and generic deterministic approach to uncertainty quantifications in non linear problems: application to fluid flow problems (2008).

[3] S.M. Adimurthi and G.V. Gowda, Conservation law with the flux function discontinuous in the space variable - II: convexconcave type fluxes and generalized entropy solutions. J. Comput. Appl. Math. 203 (2007) 310-344.

[4] S.M. Adimurthi and G.V. Gowda, Optimal entropy solutions for conservation laws with discontinuous flux-functions. J. Hyperbolic Differ. Equ. 2 (2005) 783-837.

[5] B. Andreianov, K.H. Karlsen and N.H. Risebro, A theory of $L^{1}$-dissipative solvers for scalar conservation laws with discontinuous flux. Arch. Ration. Mech. Anal. 201 (2011) 27-86. 
[6] E. Audusse and B. Perthame, Uniqueness for scalar conservation laws with discontinuous flux via adapted entropies. Proc. R. Soc. Edinburgh Sect. A: Math. 135 (2005) 253-265.

[7] J. Badwaik and A.M. Ruf, Convergence rates of monotone schemes for conservation laws with discontinuous flux. SIAM J. Numer. Anal. 58 (2020) 607-629.

[8] P. Baiti and H.K. Jenssen, Well-posedness for a class of $2 \times 2 L^{\infty}$ data. J. Differ. Equ. 140 (1997) 161-185.

[9] R. Bürger, K. Karlsen, C. Klingenberg and N. Risebro, A front tracking approach to a model of continuous sedimentation in ideal clarifier-thickener units. Nonlinear Anal.: Real World App. 4 (2003) 457-481.

[10] R. Bürger, K.H. Karlsen and J.D. Towers, An Engquist-Osher-type scheme for conservation laws with discontinuous flux adapted to flux connections. SIAM J. Numer. Anal. 47 (2009) 1684-1712.

[11] Q.-Y. Chen, D. Gottlieb and J.S. Hesthaven, Uncertainty analysis for the steady-state flows in a dual throat nozzle. J. Comput. Phys. 204 (2005) 378-398.

[12] G.M. Coclite and N.H. Risebro, Conservation laws with time dependent discontinuous coefficients. SIAM J. Math. Anal. 36 (2005) 1293-1309.

[13] S. Cox, M. Hutzenthaler, A. Jentzen, J. van Neerven and T. Welti, Convergence in Hölder norms with applications to Monte Carlo methods in infinite dimensions. IMA J. Numer. Anal. 41 (2021) 493-548.

[14] S. Diehl, A conservation law with point source and discontinuous flux function modelling continuous sedimentation. SIAM J. Appl. Math. 56 (1996) 388-419.

[15] S.S. Ghoshal, A. Jana and J.D. Towers, Convergence of a Godunov scheme to an Audusse-Perthame adapted entropy solution for conservation laws with BV spatial flux. Numer. Math. 146 (2020) 629-659.

[16] S.S. Ghoshal, J.D. Towers and G. Vaidya, Convergence of a Godunov scheme for degenerate conservation laws with BV spatial flux and a study of Panov type fluxes. Preprint: arXiv:2011.10946 (2020).

[17] S.S. Ghoshal, J.D. Towers and G. Vaidya, Well-posedness for conservation laws with spatial heterogeneities and a study of BV regularity. Preprint: arXiv:2010.13695 (2020).

[18] M. Giles, Improved multilevel Monte Carlo convergence using the Milstein scheme. In: Monte Carlo and Quasi-Monte Carlo Methods 2006. Springer (2008) 343-358.

[19] M.B. Giles, Multilevel Monte Carlo path simulation. Oper. Res. 56 (2008) 607-617.

[20] T. Gimse, Conservation laws with discontinuous flux functions. SIAM J. Math. Anal. 24 (1993) $279-289$.

[21] T. Gimse and N.H. Risebro, Riemann problems with a discontinuous flux function. In: Vol. 1 of Proceedings of Third International Conference on Hyperbolic Problems (1991) 488-502.

[22] T. Gimse and N.H. Risebro, Solution of the Cauchy problem for a conservation law with a discontinuous flux function. SIAM J. Math. Anal. 23 (1992) 635-648.

[23] T. Gimse and N.H. Risebro, A note on reservoir simulation for heterogeneous porous media. Transp. Porous Media 10 (1993) $257-270$.

[24] S. Heinrich, Multilevel Monte Carlo methods. In: International Conference on Large-Scale Scientific Computing. Springer (2001) 58-67.

[25] H. Holden and N.H. Risebro, Front Tracking for Hyperbolic Conservation Laws. Springer 152 (2015).

[26] K.H. Karlsen and J.D. Towers, Convergence of the Lax-Friedrichs scheme and stability for conservation laws with a discontinuous space-time dependent flux. Chin. Ann. Math. 25 (2004) 287-318.

[27] K.H. Karlsen and J.D. Towers, Convergence of a Godunov scheme for conservation laws with a discontinuous flux lacking the crossing condition. J. Hyperbolic Differ. Equ. 14 (2017) 671-701.

[28] K. Karlsen, N. Risebro and J. Towers, Upwind difference approximations for degenerate parabolic convection-diffusion equations with a discontinuous coefficient. IMA J. Numer. Anal. 22 (2002) 623-664.

[29] K.H. Karlsen, N.H. Risebro and J.D. Towers, $\mathrm{L}^{1}$ stability for entropy solutions of nonlinear degenerate parabolic convectiondiffusion equations with discontinuous coefficients. Preprint Series. Pure Mathematics http://urn.nb.no/URN:NBN:no-8076 (2003).

[30] C. Klingenberg and N.H. Risebro, Convex conservation laws with discontinuous coefficients. Existence, uniqueness and asymptotic behavior. Commun. Part. Differ. Equ. 20 (1995) 1959-1990.

[31] C. Klingenberg and N.H. Risebro, Stability of a resonant system of conservation laws modeling polymer flow with gravitation. J. Differ. Equ. 170 (2001) 344-380.

[32] U. Koley, N.H. Risebro, C. Schwab and F. Weber, A multilevel Monte Carlo finite difference method for random scalar degenerate convection-diffusion equations. J. Hyperbolic Differ. Equ. 14 (2017) 415-454.

[33] S.N. Kružkov, First order quasilinear equations in several independent variables. Math. USSR-Sbornik 10 (1970) $217-243$.

[34] M. Ledoux and M. Talagrand, Probability in Banach Spaces: Isoperimetry and Processes. Springer Science \& Business Media (2013).

[35] M.J. Lighthill and G.B. Whitham, On kinematic waves II. A theory of traffic flow on long crowded roads. Proc. R. Soc. London. Ser. A. Math. Phys. Sci. 229 (1955) 317-345.

[36] G. Lin, C. Su and G. Karniadakis, The stochastic piston problem. Proc. Nat. Acad. Sci. USA 101 (2004) $15840-15845$.

[37] S. Mishra, Convergence of upwind finite difference schemes for a scalar conservation law with indefinite discontinuities in the flux function. SIAM J. Numer. Anal. 43 (2005) 559-577.

[38] S. Mishra and C. Schwab, Sparse tensor multi-level Monte Carlo finite volume methods for hyperbolic conservation laws with random initial data. Math. Comput. 81 (2012) 1979-2018. 
[39] S. Mishra, C. Schwab and J. Šukys, Multi-level Monte Carlo finite volume methods for uncertainty quantification in nonlinear systems of balance laws. In: Uncertainty Quantification in Computational Fluid Dynamics. Springer (2013) 225-294.

[40] S. Mishra, D. Ochsner, A.M. Ruf and F. Weber, Bayesian inverse problems in the Wasserstein distance and application to conservation laws. in preparation (2021).

[41] S. Mishra, N.H. Risebro, C. Schwab and S. Tokareva, Numerical solution of scalar conservation laws with random flux functions. SIAM/ASA J. Uncertainty Quant. 4 (2016) 552-591.

[42] B. Piccoli and M. Tournus, A general BV existence result for conservation laws with spatial heterogeneities. SIAM J. Math. Anal. 50 (2018) 2901-2927.

[43] G. Poëtte, B. Després and D. Lucor, Uncertainty quantification for systems of conservation laws. J. Comput. Phys. 228 (2009) 2443-2467.

[44] N.H. Risebro and A. Tveito, Front tracking applied to a nonstrictly hyperbolic system of conservation laws. SIAM J. Sci. Stat. Comput. 12 (1991) 1401-1419.

[45] N.H. Risebro, C. Schwab and F. Weber, Correction to: Multilevel Monte Carlo front-tracking for random scalar conservation laws. BIT Numer. Math. 58 (2018) 247-255.

[46] A.M. Ruf, Flux-stability for conservation laws with discontinuous flux and convergence rates of the front tracking method. IMA J. Numer. Anal. 101 (2021) draa101.

[47] A.M. Ruf, E. Sande and S. Solem, The optimal convergence rate of monotone schemes for conservation laws in the Wasserstein distance. J. Sci. Comput. 80 (2019) 1764-1776.

[48] W. Shen, On the uniqueness of vanishing viscosity solutions for riemann problems for polymer flooding. Nonlinear Differ. Equ. App. NoDEA 24 (2017) 37.

[49] J. Towers, Convergence of a difference scheme for conservation laws with a discontinuous flux. SIAM J. Numer. Anal. 38 (2000) 681-698.

[50] J.D. Towers, A difference scheme for conservation laws with a discontinuous flux: the nonconvex case. SIAM J. Numer. Anal. 39 (2001) 1197-1218.

[51] J.D. Towers, An existence result for conservation laws having BV spatial flux heterogeneities - without concavity. J. Differ. Equ. 269 (2020) 5754-5764.

[52] J. Tryoen, O. Le Maitre, M. Ndjinga and A. Ern, Intrusive Galerkin methods with upwinding for uncertain nonlinear hyperbolic systems. J. Comput. Phys. 229 (2010) 6485-6511.

[53] J. Van Neerven, Stochastic evolution equations. ISEM Lecture Notes (2008).

[54] X. Wan and G.E. Karniadakis, Long-term behavior of polynomial chaos in stochastic flow simulations. Comput. Methods Appl. Mech. Eng. 195 (2006) 5582-5596.

[55] X. Wen and S. Jin, Convergence of an immersed interface upwind scheme for linear advection equations with piecewise constant coefficients I: $L^{1}$-error estimates. J. Comput. Math. 26 (2008) 1-22. 